\title{
Quantum corrections to central charges and supersymmetric Casimir energy in $\mathrm{AdS}_{3} / \mathrm{CFT}_{2}$
}

\author{
Arash Arabi Ardehali, ${ }^{a}$ Finn Larsen, ${ }^{b}$ James T. Liu ${ }^{b}$ and Phillip Szepietowski ${ }^{c}, d$ \\ ${ }^{a}$ Department of Physics and Astronomy, Uppsala University, \\ Box 516, SE-751 20 Uppsala, Sweden \\ ${ }^{b}$ Leinweber Center for Theoretical Physics, Randall Laboratory of Physics, \\ The University of Michigan, \\ Ann Arbor, MI 48109-1040, U.S.A. \\ ${ }^{c}$ Institute for Theoretical Physics, University of Amsterdam, \\ Science Park 904, 1098 XH Amsterdam, The Netherlands \\ ${ }^{d}$ Institute for Theoretical Physics and Center for Extreme Matter and Emergent Phenomena, \\ Utrecht University, \\ Princetonplein 5, 3584 CC Utrecht, The Netherlands \\ E-mail: ardehali@physics.uu.se, larsenf@umich.edu, jimliu@umich.edu, \\ p.g.szepietowski@uu.nl
}

ABStract: We study the Casimir energy of bulk fields in $\mathrm{AdS}_{3}$ and its relation to subleading terms in the central charge of the dual $\mathrm{CFT}_{2}$. Computing both sides of the standard $\mathrm{CFT}_{2}$ relation $E=-c / 12$ independently we show that this relation is not necessarily satisfied at the level of individual bulk supergravity states, but in theories with sufficient supersymmetry it is restored at the level of bulk supermultiplets. Assuming only $(0,2)$ supersymmetry (or more), we improve the situation by relating quantum corrections to the central charge and the supersymmetric Casimir energy which in turn is related to an index. These relations adapt recent progress on the $\mathrm{AdS}_{5} / \mathrm{CFT}_{4}$ correspondence to $\mathrm{AdS}_{3} / \mathrm{CFT}_{2}$ holography. We test our formula successfully in several examples, including the $(0,4) \mathrm{MSW}$ theory describing classes of $4 \mathrm{D}$ black holes and the large $(4,4)$ theory that is interesting for higher spin holography. We also make predictions for the subleading central charges in several recently proposed $(2,2)$ dualities where the $\mathrm{CFT}_{2}$ is not yet well-understood.

Keywords: AdS-CFT Correspondence, Anomalies in Field and String Theories, 1/N Expansion

ARXIV EPRINT: 1811.12367 


\section{Contents}

1 Introduction 1

2 Supersymmetric Casimir energy and the index in $\mathrm{AdS}_{5} / \mathrm{CFT}_{4} \quad 3$

3 Central charge and the Casimir energy in $\mathrm{AdS}_{3} / \mathrm{CFT}_{2} \quad 5$

3.1 The holographic Weyl anomaly 6

$\begin{array}{lll}3.2 & \text { The Casimir energy } & 7\end{array}$

3.3 Holography for various amounts of supersymmetry 9

$\begin{array}{ll}3.3 .1 \mathcal{N}=(0,2) & 9\end{array}$

$\begin{array}{lll}3.3 .2 \mathcal{N}=(2,2) & 9\end{array}$

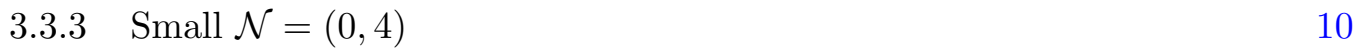

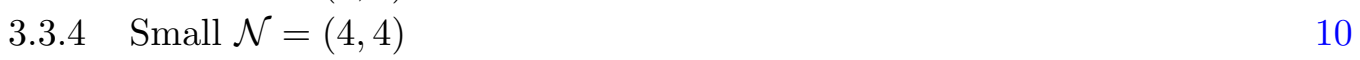

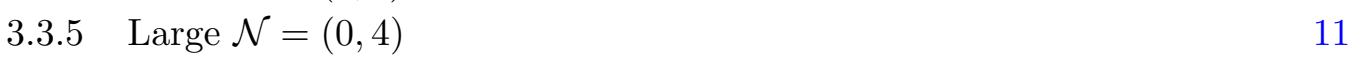

3.3.6 Large $\mathcal{N}=(4,4) \quad 12$

$\begin{array}{ll}3.4 & \text { How much supersymmetry is sufficient? } \\ \end{array}$

4 Supersymmetric Casimir energy in $\mathbf{A d S}_{3} \quad 13$

4.1 The superconformal index and the supersymmetric Casimir energy 13

$\begin{array}{lll}4.2 & \text { The single-particle index } & 15\end{array}$

$\begin{array}{ll}\text { 4.3 Quantum corrections from one-loop Chern-Simons levels } & 16\end{array}$

$\begin{array}{ll}4.4 \text { Higher amounts of supersymmetry } & 17\end{array}$

$\begin{array}{ll}\text { 4.4.1 Small } \mathcal{N}=(0,4) & 17\end{array}$

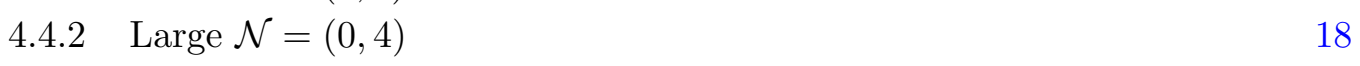

$\begin{array}{ll}4.4 .3 \mathcal{N}=(2,2) & 18\end{array}$

$\begin{array}{ll}\text { 4.4.4 Small and large } \mathcal{N}=(4,4) & 19\end{array}$

5 Quantum corrected central charge for specific dualities 20

$5.1 \mathrm{AdS}_{3} \times S^{3} \times K 3 \quad 20$

$5.2 \mathrm{AdS}_{3} \times S^{3} \times T^{4} \quad 20$

$5.3 \mathrm{AdS}_{3} \times S^{3} \times S^{3} \times S^{1} \quad 21$

5.4 M-theory on $\mathrm{AdS}_{3} \times S^{2} \times C Y_{3} \quad 22$

$5.5 \quad \mathrm{AdS}_{3} \times\left(S^{3} \times T^{4}\right) / G$ and $\mathrm{AdS}_{3} \times\left(S^{3} \times K 3\right) / \mathbb{Z}_{2}$

$5.6 \quad \mathrm{AdS}_{3} \times\left(S^{3} \times T^{4}\right) / D_{n} \quad 23$

$\begin{array}{lll}6 & \text { Summary and outlook } & 24\end{array}$

A Some 2D superconformal representation theory $\quad 26$

$\begin{array}{ll}\text { A.1 The } \mathcal{N}=2 \text { superconformal algebra } & 26\end{array}$

A.2 The small $\mathcal{N}=4$ superconformal algebra $\quad 27$

A.3 The large $\mathcal{N}=4$ superconformal algebra 28 
B Zeta function representation of the large- $c$ index and the supersymmetric Casimir energy

B.1 Supersymmetric Casimir energy from the index 30

B.2 Supersymmetric Casimir energy as a sum over normal mode energies 32

C Computation of one-loop Chern-Simons terms 33

$\begin{array}{lll}\text { C.1 Details on the vacuum polarization diagrams } & 33\end{array}$

$\begin{array}{lll}\text { C.1.1 Spin- } 1 / 2 & 33\end{array}$

$\begin{array}{lll}\text { C.1.2 Spin-1 } & 34\end{array}$

$\begin{array}{lll}\text { C.1.3 Spin-3/2 } & 34\end{array}$

C.1.4 Extrapolation to arbitrary spin 35

$\begin{array}{lll}\text { C.2 Momentum integrals } & 36\end{array}$

\section{Introduction}

More than 20 years after the advent of the AdS/CFT correspondence, "tests" of holographic duality rarely challenge the conjecture itself, but nevertheless regularly motivate investigations in quantum field theory that are interesting in their own right. Reproducing the trace anomaly presents an important "test" of holography in this sense. On the boundary, the anomalous breaking of conformal symmetry due to an inert background geometry is parametrized by central charges that are usually well understood even for finite rank $N$ of the gauge group. In the dual bulk theory the leading contribution to the anomaly for large rank $N$ of the gauge symmetry is due to a universal boundary term that is classical in nature [1]. For our purposes, the interesting bulk effect is the term that is at the subleading order in $1 / N$ and due to one-loop contributions from bulk excitations. The computation of such quantum effects is well understood in simple cases but the elaborate spectrum required for precision holography presents challenges. There has been significant progress on this problem in the case of $\mathrm{AdS}_{5} / \mathrm{CFT}_{4}$ correspondence and it has largely been solved [2-9]. The purpose of this paper is to address the situation in the case of $\mathrm{AdS}_{3} / \mathrm{CFT}_{2}$ correspondence, focusing on bulk issues.

As a matter of principle, one-loop quantum effects in the bulk theory include all modes in the theory, including modes from string theory (or some other UV completion) that might not be well-understood. Therefore, it is important to identify the settings where there is sufficient SUSY that it can be justified to ignore unknown parts of the spectrum, leaving only the known SUGRA states. Our goal is to achieve agreements in all situations where the legitimacy of focusing on SUGRA states is guaranteed by SUSY, though not necessarily in situations where SUSY is too weak, except perhaps for occasional "accidents" (that might well be due to some interesting symmetry beyond SUSY that we have not recognized).

This goal may seem modest but we identify some obstacles. A key challenge is that there is an infinite tower of Kaluza-Klein states so, effectively, the theory is higher dimensional. Renormalization of the theory in $\mathrm{AdS}_{3}$ and the subsequent sum over the KK tower does not necessarily agree with renormalization of the theory in higher dimensions. 
A specific computational scheme was introduced by Beccaria, Macorini, and Tseytlin (BMT) [10]. It amounts to computing the subleading Casimir energy $\delta E$ by summing over the ground state energy $\frac{1}{2} \omega$ for full KK towers of chiral primaries in supergravity with renormalization in the $\zeta$-function scheme, and then applying the standard $\mathrm{CFT}_{2}$ relation $\delta E=-\delta c / 12$ to extract the subleading central charge. BMT tested their prescription successfully in the cases of non-chiral theories with $(4,4)$ SUSY.

We apply the BMT prescription to compute $\delta E$ in situations with less SUSY. We also introduce an independent algorithm to compute $\delta c$ holographically so we can test the relation $\delta E=-\delta c / 12$ at the level of individual bulk representations. These tests are largely successful, but not always so.

For example, in theories with fewer than four supercharges the relation is not satisfied. However, this "failure" is not unexpected because, as we show, in this case long multiplets contribute to both $\delta E$ and $\delta c$. Unknown massive fields would be organized in such multiplets and so our supergravity computation is insufficient for an agreement. The AdS/CFT correspondence does not require agreement for individual bulk fields so, in principle, it could be that the sum over all KK fields would restore the relation $\delta E=-\delta c / 12$. However, even if such agreement could be established it would anyway be satisfying only after also explaining why long multiplets cancel between themselves. It is an additional and independent concern that the $\zeta$-function regularization may not respect supersymmetry.

To address the situation in a principled manner we consider a different approach, inspired by progress on the $\mathrm{AdS}_{5} / \mathrm{CFT}_{4}$ correspondence over the last few years. Assuming at least $(0,2)$ SUSY, one can define a right-handed superconformal index $\mathcal{I}_{\text {s.p. }}^{R}(q)$ which counts all the bulk single-particle states that are annihilated by one of the supercharges and hence vanishes when evaluated on long representations. ${ }^{1}$ The main result of the present paper is a simple relation between the high-temperature behavior of this single-particle index and the subleading central charge of the boundary CFT. More precisely, we show that the one-loop correction to the left-moving bulk central charge is given by

$$
\delta c_{L}=12 \lim _{\beta \rightarrow 0} \frac{\mathrm{d}}{\mathrm{d} \beta} \mathcal{I}_{\text {s.p. }}^{R}\left(q=e^{-\beta}\right) .
$$

The expression subject to the limit $\beta \rightarrow 0$ is a meromorphic function of $\beta$ that should be renormalized by omission of pole terms prior to the limit. An analogous expression for $\delta c_{R}$ applies to theories with $(2,0)$ SUSY.

To arrive at the formula (1.1), we leverage the notion of supersymmetric Casimir energy $E_{S U S Y}^{L}$, related to $c_{L}$ via $E_{S U S Y}^{L}=-c_{L} / 24$. That a supersymmetric version of the Casimir energy can be defined from the superconformal index was first proposed in $[11,12]$ at the level of single-letter indices in free field theory. We apply this idea to the bulk single-particle index in order to extract the subleading $E_{S U S Y}^{L}$, and hence the central charge $\delta c_{L}$.

We successfully test the simple relation (1.1) in section 5, in the context of several well-known $\mathrm{AdS}_{3} / \mathrm{CFT}_{2}$ dualities. We also make predictions for the subleading central charges in several other cases where the bulk index is known but the boundary CFT is not.

\footnotetext{
${ }^{1}$ The superconformal index is essentially the spectral flow of the elliptic genus to the NS-NS sector; see section 4 for the explicit definitions.
} 
This paper is organized as follows. In section 2 we review some aspects of the supersymmetric Casimir energy $E_{S U S Y}$ that were previously developed in the context of the $\mathrm{AdS}_{5} / \mathrm{CFT}_{4}$ correspondence. It allows a concise expression of the bulk quantum corrections to the Weyl anomaly coefficients $a$ and $c$ obtained in [7,9]. This will serve as an introduction to the techniques we employ in $\mathrm{AdS}_{3} / \mathrm{CFT}_{2}$ holography and motivate our formula (1.1). In section 3 we discuss possible relations between the bulk Casimir energy of supergravity states in $\mathrm{AdS}_{3}$ and the central charge of the dual 2D CFT. We refine the discussion by defining left-moving and right-moving Casimir energies and attempt to relate these to the corresponding left and right-moving central charges, emphasizing the relevance of a supersymmetric spectrum to the success or failure of such relations at the level of individual bulk multiplets. In section 4 we derive the supersymmetric Casimir energy from the superconformal index and compute from it the left-moving central charge of $\mathcal{N}=(0,2)$ theories. In order to provide a consistency check of our approach in this minimal supersymmetric context we additionally find the subleading right-moving central charge $\delta c_{R}$ by computing one-loop Chern-Simons levels $\delta k_{R}$ for the $\mathrm{U}(1)_{R}$ gauge field, which are related by $\delta c_{R}=-3 \delta k_{R}$. We also discuss analogous relations in theories with more supercharges. In section 5 we test the results from section 4 in particular examples of $\mathrm{AdS}_{3} / \mathrm{CFT}_{2}$ duality, including the $(0,4) \mathrm{MSW}$ theory describing classes of $4 \mathrm{D}$ black holes and the large $(4,4)$ theory that is interesting for higher spin holography. We also offer predictions for quantum corrections to the central charge in several $(2,2)$ cases where the supersymmetric index of bulk supergravity states is known but the dual $\mathrm{CFT}_{2}$ is not. We conclude with a summary and a discussion of outlooks in section 6 .

\section{Supersymmetric Casimir energy and the index in $\mathrm{AdS}_{5} / \mathrm{CFT}_{4}$}

Before turning to $\mathrm{AdS}_{3} / \mathrm{CFT}_{2}$ and the justification of the relation (1.1) it is instructive to review the $\mathrm{AdS}_{5} / \mathrm{CFT}_{4}$ case.

The supersymmetric partition function $Z_{S U S Y}$, defined as the path integral with appropriate boundary conditions, is proportional to the index $\mathcal{I}$, defined in terms of a trace over the states that respect supersymmetry. The factor of proportionality defines the supersymmetric Casimir energy $E_{\mathrm{SUSY}}$ through [11-16].

$$
Z_{\mathrm{SUSY}}=e^{-\beta E_{\mathrm{SUSY}}} \mathcal{I} \text {. }
$$

In view of its definition, $E_{\text {SUSY }}$ plays well with supersymmetry, while the conventional Casimir energy might not.

Like the Casimir energy in 2D, the supersymmetric Casimir energy in $4 \mathrm{D}$ is related to the central charges of the theory. Specifically, it can be written in terms of the Weyl anomaly coefficients $a$ and $c$ of an $\mathcal{N}=1$ SCFT as [13-16]

$$
E_{\mathrm{SUSY}}=\frac{8}{3}\left(b_{1}+b_{2}\right)(a-c)+\frac{8}{27} \frac{\left(b_{1}+b_{2}\right)^{3}}{b_{1} b_{2}}(3 c-2 a) .
$$

Here $b_{1}$ and $b_{2}$ parametrize families of supersymmetric boundary conditions or, equivalently, the fugacities $t=e^{\left(b_{1}+b_{2}\right) \beta}$ and $y=e^{\left(b_{1}-b_{2}\right) \beta}$ that appear in the superconformal index introduced in (2.6) below. 
The leading semi-classical saddle point determines central charges of $\mathcal{O}\left(N^{2}\right)$. From the bulk point of view the subleading $\mathcal{O}(1)$ contributions are quantum corrections. The superconformal index provides a useful regulator for determining these quantum corrections. In particular, the subleading central charges - given below in (2.3) - can be computed by extracting the subleading supersymmetric Casimir energy from the single-trace superconformal index $[7,9]$.

In $\mathrm{AdS}_{5}$, bulk excitations can be organized into multiplets of the superconformal algebra. Representations are labeled by the conformal dimension $\Delta$, a $\mathrm{U}(1)_{R}$ charge $r$, and two $\mathrm{SU}(2)$ spins $j_{1}$ and $j_{2}$. The central charges are only quantum corrected by loops of shortened multiplets $[5,8]$. The contribution from a single short multiplet is given by $[6,8]$

$$
\begin{aligned}
c_{\text {chiral }}=-c_{\text {SLII }}= & (-1)^{2 j_{1}+2 j_{2}} \frac{9}{128}\left(2 j_{1}+1\right)\left(\Delta-\frac{r}{2}-1\right) \\
& \times\left[\left(\Delta-\frac{r}{2}\right)\left(\Delta-\frac{r}{2}-2\right)+\frac{4}{9} j_{1}\left(j_{1}+1\right)+\frac{4}{9}\right], \\
a_{\text {chiral }}=-a_{\text {SLII }}= & (-1)^{2 j_{1}+2 j_{2}} \frac{9}{128}\left(2 j_{1}+1\right)\left(\Delta-\frac{r}{2}-1\right) \\
& \times\left[\left(\Delta-\frac{r}{2}\right)\left(\Delta-\frac{r}{2}-2\right)-\frac{4}{3} j_{1}\left(j_{1}+1\right)+\frac{2}{3}\right],
\end{aligned}
$$

where chiral multiplets satisfy the shortening condition

$$
\Delta=\frac{3 r}{2}, \quad j_{2}=0
$$

and semi-long II (SLII) multiplets satisfy

$$
\Delta=\frac{3 r}{2}+2 j_{2}+2
$$

Multiplets with conserved currents are included as the special case of SLII multiplets with the additional condition $\frac{3}{2} r-j_{1}-j_{2}=0$. The fact that the contributions are equal and opposite for the two types of shortened multiplets is consistent with the recombination rules for forming a long multiplet out of a chiral and an SLII multiplet along with the fact that long multiplets do not contribute to the central charges. The central charges also receive contributions from anti-chiral and SLI multiplets; these are given by (2.3) with the replacements $j_{1} \leftrightarrow j_{2}$ and $r \rightarrow-r$.

The right-handed $\mathcal{N}=1$ superconformal index is given by the trace

$$
\mathcal{I}^{R}(t, y)=\operatorname{Tr}(-1)^{F} t^{-\left(\Delta-\frac{r}{2}\right)} y^{2 j_{1}} .
$$

This only receives contributions from states which satisfy $\Delta-\frac{3}{2} r-2 j_{2}=0$. As these states are not present in long multiplets, the index only receives contributions from shortened representations of the superconformal algebra. Henceforth we will drop the $R$ superscript. Analogous results for the left-handed index follow from the replacements $j_{1} \leftrightarrow j_{2}$ and $r \rightarrow-r$.

The theories we consider are effectively free at large $N$, so multiparticle states are generated from single particle states by simple combinatorics. In $\mathrm{AdS}_{5} / \mathrm{CFT}_{4}$ this means 
we can consider single-particle states in AdS which are dual to single-trace operators in the CFT. In this case the full multi-particle index $\mathcal{I}(t, y)$ is simply related to the single-particle index by means of the plethystic exponential

$$
\mathcal{I}(t, y)=\exp \left(\sum_{k=1}^{\infty} \frac{1}{k} \mathcal{I}_{\text {s.p. }}\left(t^{k}, y^{k}\right)\right),
$$

where the single-particle index is given by the same trace in (2.6), except restricted to single-particle states

$$
\mathcal{I}_{\text {s.p. }}(t, y)=\operatorname{Tr}_{\text {s.p. }}(-1)^{F} t^{-\left(\Delta-\frac{r}{2}\right)} y^{2 j_{1}} .
$$

The single-particle superconformal index for individual short multiplets is

$$
\mathcal{I}_{s . p .}^{\text {chiral }}(t, y)=-\mathcal{I}_{s . p .}^{\text {SLII }}(t, y)=(-1)^{2\left(j_{1}+j_{2}\right)} \frac{t^{-\left(\Delta-\frac{r}{2}\right)} \chi_{j_{1}}(y)}{\left(1-t^{-1} y\right)\left(1-t^{-1} y^{-1}\right)} .
$$

where

$$
\chi_{j}(y)=\frac{y^{j+\frac{1}{2}}-y^{-j-\frac{1}{2}}}{y^{\frac{1}{2}}-y^{-\frac{1}{2}}}
$$

is the $\mathrm{SU}(2)$ character.

The free-field description allows us to compute the supersymmetric Casimir energy by extracting the term that is linear in $\beta$ from the single-particle index. In particular, by inserting the index for a shortened multiplet given in (2.9) into

$$
E_{\mathrm{SUSY}}=-\left.\frac{1}{2} \lim _{\beta \rightarrow 0} \partial_{\beta} \mathcal{I}_{\text {s.p. }}\left(t=e^{\left(b_{1}+b_{2}\right) \beta}, y=e^{\left(b_{1}-b_{2}\right) \beta}\right)\right|^{\text {finite }} .
$$

we recover the result in (2.2) with $a$ and $c$ given by $(2.3) .^{2}$ Thus we can utilize the superconformal index as a method of computing the central charges. This is the main idea that we will adapt to the context of $\mathrm{AdS}_{3} / \mathrm{CFT}_{2}$ in section 4.

The result in (2.10) can alternatively be understood as a regularized sum of supersymmetric energies of states $[11,12]$, with the derivative with respect to $\beta$ in $(2.10)$ bringing down the supersymmetric energy of a single-particle state. In appendix B we discuss the relation between this type of regularization and the zeta-function regularization in the context of $\mathrm{AdS}_{3} / \mathrm{CFT}_{2}$. This explains why the term in the single-particle index that is linear in $\beta$ is equivalent to the $\mathcal{O}(1)$ contribution to the supersymmetric Casimir energy as defined in (2.1) for large- $N$ theories.

\section{Central charge and the Casimir energy in $\mathrm{AdS}_{3} / \mathrm{CFT}_{2}$}

In two-dimensional conformal field theories, the standard (i.e. non-supersymmetric) Casimir energy is simply related to the central charge by $[17,18]$

$$
E_{c}=-\frac{c}{12} \text {. }
$$

\footnotetext{
${ }^{2}$ For the precise numerical agreement note that the Casimir energy in (2.2) is written in terms of the full central charge, which receives contributions also from anti-chiral and SLI multiplets. These satisfy $a_{\text {chiral }}(\Delta, j, 0, r)=a_{\text {anti-chiral }}(\Delta, 0, j,-r)$ and $a_{\text {SLII }}\left(\Delta, j_{1}, j_{2}, r\right)=a_{\text {SLI }}\left(\Delta, j_{2}, j_{1},-r\right)$ so they introduce an overall factor of two.
} 
In this section, we explore the connection between these two quantities from a holographic point of view. As in the previous section, our focus is on the $\mathcal{O}(1)$ contributions arising from one-loop effects in the bulk. As reviewed below, both quantities can be obtained from the one-loop partition function, which can be decomposed as a sum over the Kaluza-Klein spectrum. From this point of view, it was noted in [10] that the Casimir energy/central charge relation does not generally hold for the holographic contribution of individual states in the bulk. Nevertheless, since (3.1) must hold for all two-dimensional conformal field theories, it must somehow be recovered after summing over the Kaluza-Klein tower.

Some of the best developed examples of $\mathrm{AdS}_{3} / \mathrm{CFT}_{2}$ duality have $\mathcal{N}=(4,4)$ supersymmetry and [10] found that, with this much supersymmetry, the relation (3.1) does hold, after all. It is satisfied on an individual multiplet by multiplet basis, even before the sum over the Kaluza-Klein states is taken. However, $\mathrm{AdS}_{3} / \mathrm{CFT}_{2}$ correspondence applies more generally and it is interesting to find criteria for the viability of a holographic description. Thus we explore theories with various amounts of supersymmetry, starting with $\mathcal{N}=(0,2)$.

\subsection{The holographic Weyl anomaly}

The holographic Weyl anomaly encoded in the leading central charge $c^{(0)}$ can be obtained from the logarithmically divergent part of the bulk action [1]. For our purposes it is convenient to express the result as $[19,20]$

$$
c^{(0)}=6 \pi \ell^{3} \frac{I_{\mathrm{on}-\text { shell }}}{\operatorname{vol}\left(A d S_{3}\right)},
$$

where $I_{\text {on-shell }}$ is the classical action

$$
I=\frac{1}{16 \pi G_{3}} \int \sqrt{g} \mathcal{L}_{3}
$$

evaluated on-shell. For Einstein gravity with a cosmological constant the on-shell Lagrangian is given by $\mathcal{L}_{3}=-R+2 \Lambda=4 / \ell^{2}$, which yield the Brown-Henneaux central charge $c^{(0)}=3 \ell / 2 G_{3}$.

We can generalize the classical bulk result to include quantum corrections by modifying the action $I \rightarrow I+\delta I_{\text {eff }}$ so that the central charge receives a correction

$$
\delta c=6 \pi \ell^{3} \frac{\delta I_{\mathrm{eff}}}{\operatorname{vol}\left(A d S_{3}\right)} .
$$

Here we take the one-loop effective action

$$
\delta I_{\mathrm{eff}}=(-1)^{2 s} \frac{1}{2} \log \operatorname{det} D
$$

where $s$ is the spin and $D$ is the appropriate differential operator appearing in the bulk field's kinetic term. The statistical factor $(-1)^{2 s}$ takes into account the fact that fermionic and bosonic determinants go in the numerator and denominator of the partition function, respectively. 
The one-loop effective action is divergent, and can be regulated by the spectral zeta function. For an $\mathrm{AdS}_{3}$ representation labeled by $\mathrm{SO}(2,2)$ quantum numbers $(\Delta, s)$ we find

$$
\begin{aligned}
\delta I_{\text {eff }} & =-\frac{1}{2}(-1)^{2 s} \zeta^{\prime}(0, D) \\
& =\frac{1}{6}(-1)^{2 s} \nu\left(\nu^{2}-3 s^{2}\right) \log R,
\end{aligned}
$$

where $\nu=\Delta-1$, and $R$ is a cut-off scale regularizing the $\mathrm{AdS}_{3}$ volume. In going to the second line we have used the result of [21] (their eq. (3.8)) for the spectral zeta function. Combining (3.4) with (3.6), and using the regulated $\operatorname{vol}\left(A d S_{3}\right)=-2 \ell^{3} \pi \log R$, we arrive at $[10]^{3}$

$$
\delta c(\Delta, s)=-\frac{1}{2}(-1)^{2 s} \nu\left(\nu^{2}-3 s^{2}\right) \quad(\nu=\Delta-1) .
$$

We may also translate the $\operatorname{AdS}_{3}$ labels $(\Delta, s)$ to the equivalent CFT weights $h$ and $\bar{h}$, so that

$$
\delta c(h, \bar{h})=-\frac{1}{2}(-1)^{2 s} \nu\left(\nu^{2}-3 s^{2}\right) \quad(\nu=h+\bar{h}-1, \quad s=h-\bar{h}) .
$$

Finally, for massless bulk fields which have $\bar{h}$ (resp. $h$ ) equal to zero, a further ghost contribution should be included so that the combined contribution to $\delta c_{\text {massless }}$ is $\delta c(h, 0)-$ $\delta c(h, 1)(\operatorname{resp} . \delta c(0, \bar{h})-\delta c(1, \bar{h}))[22]$.

\subsection{The Casimir energy}

Turning now to the Casimir energy, we take a 2D CFT point of view and start with the partition function

$$
Z(q, \bar{q})=\operatorname{Tr}(-1)^{F_{L}+F_{R}} q^{L_{0}} \bar{q}^{\bar{L}_{0}},
$$

where $L_{0}$ and $\bar{L}_{0}$ are left and right Virasoro generators and the total fermion number can be replaced with the spin of the representation as $(-1)^{F_{L}+F_{R}}=(-1)^{2 s}=(-1)^{2\left(L_{0}-\bar{L}_{0}\right)}$. For free fields we can then formally define

$$
E_{c}=\frac{1}{2} \operatorname{Tr}(-1)^{F}\left(L_{0}+\bar{L}_{0}\right)=\left.\frac{1}{2} q \frac{d}{d q} Z(q, \bar{q}=q)\right|_{q \rightarrow 1} .
$$

The idea expressed by the formula is very basic, a generalization of the ground state energy $\frac{1}{2} \hbar \omega$ for the harmonic oscillator. However, as always in quantum field theory, we must address divergences. We find it convenient to introduce a regulator through the substitution $q=e^{-\epsilon}$ which renders (3.10) a meromorphic function of $\epsilon$. We subsequently take the limit $q \rightarrow 1$ by retaining only the constant term in the Laurent expansion around $\epsilon=0$. As a concise shorthand for this procedure we write:

$$
E_{c}=-\left.\frac{1}{2} \lim _{\epsilon \rightarrow 0} \frac{d}{d \epsilon} Z\left(e^{-\epsilon}, e^{-\epsilon}\right)\right|^{\text {finite }} .
$$

This prescription for extracting the Casimir energy is equivalent to that defined via the spectral zeta function. Indeed, following [10], one defines

$$
E_{c}=\frac{1}{2}(-1)^{2 s} \sum_{n}\left(h_{n}+\bar{h}_{n}\right)=\frac{1}{2}(-1)^{2 s} \zeta(-1)
$$

\footnotetext{
${ }^{3}$ Note that our $\delta c$ is denoted in [10] by $c_{\mathrm{AdS}_{3}}^{+}$.
} 
where $h_{n}$ and $\bar{h}_{n}$ are eigenvalues of $L_{0}$ and $\bar{L}_{0}$ and

$$
\zeta(z)=\sum_{n} \frac{1}{\left(h_{n}+\bar{h}_{n}\right)^{z}}=\frac{1}{\Gamma(z)} \int_{0}^{\infty} d \beta \beta^{z-1} Z\left(e^{-\beta}, e^{-\beta}\right) .
$$

One can refine (3.10) by taking independent $q$ and $\bar{q}$ derivatives of the partition function. In this way, we may define the "left" and "right" contributions to the Casimir energy

$$
\begin{aligned}
& E_{c}^{L}=-\left.\frac{1}{2} \lim _{\epsilon \rightarrow 0}\left(\frac{d}{d \epsilon} Z\left(e^{-\epsilon}, \bar{q}\right)\right)_{\bar{q}=e^{-\epsilon}}\right|^{\text {finite }}, \\
& E_{c}^{R}=-\left.\frac{1}{2} \lim _{\epsilon \rightarrow 0}\left(\frac{d}{d \epsilon} Z\left(q, e^{-\epsilon}\right)\right)_{q=e^{-\epsilon}}\right|^{\text {finite }} .
\end{aligned}
$$

In these two formulae the large round brackets serve to stress that the finite substitutions $\bar{q} \rightarrow e^{-\epsilon}$ and $q \rightarrow e^{-\epsilon}$ are performed after differentiation with respect to $\epsilon$ but before extracting the constant part of the expressions from the Laurent expansion in $\epsilon$ around $\epsilon=0$. The prescriptions for $E_{c}^{L}$ and $E_{c}^{R}$ in (3.14) and (3.15) can also be extracted from the Casimir energy computed from a suitably refined zeta function. However, they do not themselves correspond precisely to the Casimir energy and are instead given by particular derivatives of the refined Casimir energy. Therefore, while it is tempting to relate $E_{c}^{L}$ and $E_{c}^{R}$ to the left and right-moving central charges, it is not clear that such an identification is justified. However, it follows straightforwardly from the chain rule that the sum

$$
E_{c}=E_{c}^{L}+E_{c}^{R}
$$

reproduces the physical Casimir energy.

Until now, we have not yet specified the one-particle partition function $Z(q, \bar{q})$. For a bulk field dual to an operator with weights $h$ and $\bar{h}$, it takes the simple form

$$
Z_{h, \bar{h}}(q, \bar{q})=\frac{q^{h} \bar{q}^{\bar{h}}}{(1-q)(1-\bar{q})},
$$

where the denominator arises from the $\mathrm{SL}(2, \mathbb{R}) \times \mathrm{SL}(2, \mathbb{R})$ descendents of the highest weight state. Performing the manipulations above, we find

$$
\begin{aligned}
& E_{c}^{L}(h, \bar{h})=-\frac{1}{48}(-1)^{2(h-\bar{h})}\left(1-10 h+18 h^{2}-8 h^{3}+12 h \bar{h}-12 h^{2} \bar{h}-6 \bar{h}^{2}+4 \bar{h}^{3}\right), \\
& E_{c}^{R}(h, \bar{h})=-\frac{1}{48}(-1)^{2(h-\bar{h})}\left(1-10 \bar{h}+18 \bar{h}^{2}-8 \bar{h}^{3}+12 h \bar{h}-12 h \bar{h}^{2}-6 h^{2}+4 h^{3}\right) .
\end{aligned}
$$

Adding these together and replacing $h=\frac{1}{2}(\nu+s+1)$ and $\bar{h}=\frac{1}{2}(\nu+1-s)$ then gives [10]

$$
E_{c}(h, \bar{h})=\frac{1}{24}(-1)^{2 s} \nu\left(2 \nu^{2}-1\right) \quad(\nu=h+\bar{h}-1, \quad s=h-\bar{h}) .
$$

As noted in [10], the central charge expression (3.8) and the Casimir energy (3.19) in general do not obey the two-dimensional CFT relation $E_{c}=-c / 12$. This is perhaps an unusual aspect of $\mathrm{AdS}_{3} / \mathrm{CFT}_{2}$ holography, and we will explore this connection in more detail below. To do so, we find it convenient to define the would-be central charge

$$
\delta \tilde{c} \equiv-12 E_{c}=-12\left(E_{c}^{L}+E_{c}^{R}\right),
$$

obtained from the Casimir energy. Below, we examine the relation between these two notions of the central charge for theories with varying amounts of supersymmetry. 


\begin{tabular}{|c|ll|}
\hline $\mathcal{N}=(0,2)$ & long $(\bar{h}>|\bar{r}|)$ & short $(\bar{h}=|\bar{r}|)$ \\
\hline$E_{c}^{L}$ & $-\frac{1}{8} \bar{h}$ & $-\frac{1}{48}\left(1-6 h+6 h^{2}+3 \bar{h}-6 \bar{h}^{2}\right)$ \\
$E_{c}^{R}$ & $-\frac{1}{16}(1-2 h-4 \bar{h})$ & $-\frac{1}{32}\left(1-8 \bar{h}+8 \bar{h}^{2}-2 h+8 h \bar{h}\right)$ \\
$\delta \tilde{c}$ & $\frac{3}{4}(1-2 h-2 \bar{h})$ & $\frac{1}{8}\left(5+12 h^{2}+12 \bar{h}^{2}-18 h-18 \bar{h}+24 h \bar{h}\right)$ \\
\hline$\delta c$ & $\frac{3}{4}(1-2 h+2 \bar{h})$ & $\frac{1}{8}\left(5+12 h^{2}-12 \bar{h}^{2}-18 h-6 \bar{h}+24 h \bar{h}\right)$ \\
\hline
\end{tabular}

Table 1. The left and right Casimir energies, $E_{c}^{L}$ and $E_{c}^{R}$, would-be central charge $\delta \tilde{c}$ and central charge $\delta c$ for $\mathcal{N}=(0,2)$ multiplets. Note that $\delta \tilde{c} \neq \delta c$ for both short and long multiplets. All entries should be multiplied by the spin-dependent factor $(-1)^{2(h-\bar{h})}$.

\subsection{Holography for various amounts of supersymmetry}

The finite-dimensional subgroup $\mathrm{SO}(2,2)$ of the two-dimensional conformal group splits into left and right components, $\mathrm{SU}(1,1) \times \mathrm{SU}(1,1)$. This can be extended by including varying amounts of supersymmetry on the left and the right, independently. We now turn to a few important cases.

\subsection{1 $\mathcal{N}=(0,2)$}

The $\mathcal{N}=2$ superconformal algebra corresponds to $\mathrm{SU}(1,1 \mid 1)$, and extends the conformal algebra formed by $L_{0}, L_{ \pm 1}$ with the supercurrents $G_{ \pm 1 / 2}^{ \pm}$and a $\mathrm{U}(1)$ current $J_{0}$. Lowest weight representations are labeled by the weight $h$ and charge $r$, and unitary representations exist for $h \geq|r|$. Such representations fall into two categories, namely long for $h>|r|$ and short for $h=|r|$. The latter may be classified as either chiral for $h=r$ or anti-chiral for $h=-r$. The content of these representations are given by

$$
\begin{array}{rlrl}
\text { long: } & & |h, r\rangle & \oplus\left|h+\frac{1}{2}, r+\frac{1}{2}\right\rangle \oplus\left|h+\frac{1}{2}, r-\frac{1}{2}\right\rangle \oplus|h+1, r\rangle, \\
\text { chiral: } & |h, h\rangle \oplus\left|h+\frac{1}{2}, h-\frac{1}{2}\right\rangle, & \\
\text { antichiral: } & |h,-h\rangle \oplus\left|h+\frac{1}{2}, \frac{1}{2}-h\right\rangle .
\end{array}
$$

For the $\mathcal{N}=(0,2)$ case, we take a single irreducible representation $|h\rangle$ on the left and tensor it with $|\bar{h}, \bar{r}\rangle$ on the right. The resulting representations are then classified by the right-moving superalgebra, and the result for the Casimir energies and central charge are shown in table 1 . As shown in the table, the would-be central charge $\delta \tilde{c}$ computed from the Casimir energy does not agree with the holographic calculation of $\delta c$.

\subsection{2 $\mathcal{N}=(2,2)$}

Although $\mathcal{N}=(0,2)$ supersymmetry is insufficient to give agreement between $\delta \tilde{c}$ and $\delta c$, it turns out that a match is obtained for $\mathcal{N}=(2,2)$ superconformal theories. Here, since both sides are supersymmetric, we may tensor together either long or short multiplets on both sides. This gives rise to the four possibilities shown in table 2. Importantly, the longlong representations do not contribute to either the Casimir energy nor the central charge. Moreover, $E_{c}^{L}$ only receives contributions from shortened representations on the right, 


\begin{tabular}{|c|llll|}
\hline $\mathcal{N}=(2,2)$ & long-long & long-short & short-long & short-short \\
\hline$E_{c}^{L}$ & 0 & $-\frac{1}{16}$ & 0 & $-\frac{1}{32}(1-4 h)$ \\
$E_{c}^{R}$ & 0 & 0 & $-\frac{1}{16}$ & $-\frac{1}{32}(1-4 \bar{h})$ \\
$\delta \tilde{c}$ & 0 & $\frac{3}{4}$ & $\frac{3}{4}$ & $\frac{3}{4}(1-2 h-2 \bar{h})$ \\
\hline$\delta c$ & 0 & $\frac{3}{4}$ & $\frac{3}{4}$ & $\frac{3}{4}(1-2 h-2 \bar{h})$ \\
\hline
\end{tabular}

Table 2. The left and right Casimir energies, $E_{c}^{L}$ and $E_{c}^{R}$, would-be central charge $\delta \tilde{c}$ and central charge $\delta c$ for $\mathcal{N}=(2,2)$ multiplets. All multiplets have $\delta \tilde{c}=\delta c$. All entries should be multiplied by the spin-dependent factor $(-1)^{2(h-\bar{h})}$.

while $E_{c}^{R}$ only receives contributions from shortened representations on the left. The fact that short representations suffice for certain computations underpins our ability to employ two-dimensional superconformal indices for efficiently packaging those computations below.

\subsubsection{Small $\mathcal{N}=(0,4)$}

We now turn to theories with $\mathcal{N}=4$ supersymmetry. Here we have to make a distinction between the "small" and the "large" $\mathcal{N}=4$. The small algebra contains $\operatorname{PSU}(1,1 \mid 2)$ as its finite dimensional subalgebra and the large $\mathcal{A}_{\gamma}$ contains $D(2,1 \mid \alpha)$.

We start with the small $\mathcal{N}=4$ algebra, with the finite dimensional subalgebra generated by $L_{0}, L_{ \pm 1}, \mathrm{SU}(2)$ currents $J_{0}^{i}$ and supercurrents $G_{ \pm 1 / 2}^{a}, \tilde{G}_{ \pm 1 / 2}^{a}$ transforming as doublets under $\mathrm{SU}(2)$. In addition, the central charge is related to the level $k$ of the affine $\mathrm{SU}(2)$ via $c=6 k$.

Lowest weight representations of the small $\mathcal{N}=4$ algebra are built from a state $|h, j\rangle$, where $j$ now labels the $\mathrm{SU}(2)$ representation. Once again, unitary representations exist for $h \geq j$, with saturation of the inequality corresponding to shortened representations. Complete representations for the small $\mathcal{N}=(0,4)$ case are then obtained by tensoring a representation $|h\rangle$ on the left with an $\mathcal{N}=4$ representation $|\bar{h}, \bar{j}\rangle$ on the right. The resulting representations are short for $\bar{h}=\bar{j}$ and long for $\bar{h}>\bar{j}$.

The resulting Casimir energies and central charges are given in table 3 . We find that the small $\mathcal{N}=(0,4)$ supersymmetry is sufficient for the would-be central charge derived from the Casimir energy $\delta \tilde{c}$ to coincide with the holographic central charge $\delta c$.

\subsubsection{Small $\mathcal{N}=(4,4)$}

Turning to the small $\mathcal{N}=(4,4)$ case, we can build representations by tensoring together either long or short $\mathcal{N}=4$ representations on the left and on the right. Since the Casimir energy and central charge vanishes identically whenever there is an $\mathcal{N}=4$ long representation, whether on the left or the right, only the short-short case will contribute non-trivially. The results are summarized in table 4 . The short-short result can also be obtained by decomposing the multiplet into $\mathcal{N}=(2,2)$ representations and using the results shown in table 2. Once again, we find that there is sufficient supersymmetry that $\delta \tilde{c}=\delta c$. This is the result previously reported in [10]. 


\begin{tabular}{|c|ll|}
\hline \multicolumn{2}{|c|}{ small } & \\
$\mathcal{N}=(0,4)$ & long $(\bar{h}>\bar{j})$ & short $(\bar{h}=\bar{j})$ \\
\hline$E_{c}^{L}$ & 0 & $-\frac{1}{24}\left(1-6 h+6 h^{2}\right)$ \\
$E_{c}^{R}$ & 0 & $\frac{1}{8} \bar{h}(1-2 h)$ \\
$\delta \tilde{c}$ & 0 & $\frac{1}{2}\left(1+6 h^{2}+6 h \bar{h}-6 h-3 \bar{h}\right)$ \\
\hline$\delta c$ & 0 & $\frac{1}{2}\left(1+6 h^{2}+6 h \bar{h}-6 h-3 \bar{h}\right)$ \\
\hline
\end{tabular}

Table 3. The left and right Casimir energies, $E_{c}^{L}$ and $E_{c}^{R}$, would-be central charge $\delta \tilde{c}$ and central charge $\delta c$ for small $\mathcal{N}=(0,4)$ multiplets labeled by $|h ; \bar{h}, \bar{j}\rangle$. All multiplets have $\delta \tilde{c}=\delta c$. All entries should be multiplied by the spin-dependent factor $(-1)^{2(h-\bar{h})}$.

\begin{tabular}{|c|llll|}
\hline \multicolumn{1}{|c|}{ small } & & & & \\
$\mathcal{N}=(4,4)$ & long-long & long-short & short-long & short-short \\
\hline$E_{c}^{L}$ & 0 & 0 & 0 & $\frac{1}{4} h$ \\
$E_{c}^{R}$ & 0 & 0 & 0 & $\frac{1}{4} \bar{h}$ \\
$\delta \tilde{c}$ & 0 & 0 & 0 & $-3(h+\bar{h})$ \\
\hline$\delta c$ & 0 & 0 & 0 & $-3(h+\bar{h})$ \\
\hline
\end{tabular}

Table 4. The left and right Casimir energies, $E_{c}^{L}$ and $E_{c}^{R}$, would-be central charge $\delta \tilde{c}$ and central charge $\delta c$ for small $\mathcal{N}=(4,4)$ multiplets. All multiplets have $\delta \tilde{c}=\delta c$. A ll entries should be multiplied by the spin-dependent factor $(-1)^{2(h-\bar{h})}$.

\subsubsection{Large $\mathcal{N}=(0,4)$}

We now consider the large $\mathcal{N}=4$ cases. The large $\mathcal{N}=4$ superalgebra contains, in addition to Virasoro, two affine $\mathrm{SU}(2)$ 's and a $\mathrm{U}(1)$ algebra. The fermionic generators include dimension-3/2 supercurrents and dimension- $1 / 2$ fields, both transforming as $(2,1)+(1,2)$ under the two $\mathrm{SU}(2)$ 's. The two affine $\mathrm{SU}(2)$ 's have levels $k^{+}$and $k^{-}$, and the central charge is given by $c=6 k^{+} k^{-} /\left(k^{+}+k^{-}\right)$. It is customary to define the parameters

$$
\gamma=\frac{k^{-}}{k^{+}+k^{-}}, \quad \alpha=\frac{\gamma}{1-\gamma}=\frac{k^{-}}{k^{+}}
$$

For a holographic point of view, we are mostly interested in the finite subalgebra $D(2,1 \mid \alpha)$ with bosonic component $\mathrm{SL}(2 ; \mathbb{R}) \times \mathrm{SU}(2) \times \mathrm{SU}(2)$. It admits unitary representations whenever [23]

$$
h \geq \gamma j^{+}+(1-\gamma) j^{-}
$$

Once again, representations split into long and short, with the latter saturating this bound. Using (3.18) and (3.8), we then arrive at the results shown in table 5. We see that generally the large $\mathcal{N}=(0,4)$ is insufficient to ensure $\delta \tilde{c}=\delta c$ even though it has four supersymmetries. However, the equality applies for some representations, notably those with $\bar{j}^{+}=\bar{j}^{-}$. 


\begin{tabular}{|c|ll|}
\hline \multicolumn{2}{|c|}{ large } \\
$\mathcal{N}=(0,4)$ & long & short \\
\hline$E_{c}^{L}$ & 0 & $-\frac{1}{24}\left[1-6 h+6 h^{2}+6 \gamma(1-\gamma)\left(\bar{j}^{+}-\bar{j}^{-}\right)^{2}\right]$ \\
$E_{c}^{R}$ & 0 & $-\frac{1}{8}\left(\bar{j}^{+}-\bar{j}^{-}\right)\left[(1-2 \gamma)(1-2 h)-4 \gamma(1-\gamma)\left(\bar{j}^{+}-\bar{j}^{-}\right)\right]$ \\
$\delta \tilde{c}$ & 0 & $\frac{1}{2}\left[1+6 h^{2}-6 h+3\left(\bar{j}^{+}-\bar{j}^{-}\right)\left((1-2 \gamma)(1-2 h)-2 \gamma(1-\gamma)\left(\bar{j}^{+}-\bar{j}^{-}\right)\right)\right]$ \\
\hline$\delta c$ & 0 & $\frac{1}{2}\left[1+6 h^{2}-6 h+3\left(\bar{j}^{+}-\bar{j}^{-}\right)\left((1-2 \gamma)(1-2 h)+2 \gamma(1-\gamma)\left(\bar{j}^{+}-\bar{j}^{-}\right)\right)\right]$ \\
\hline
\end{tabular}

Table 5. The left and right Casimir energies, $E_{c}^{L}$ and $E_{c}^{R}$, would-be central charge $\delta \tilde{c}$ and central charge $\delta c$ for large $\mathcal{N}=(0,4)$ multiplets labeled by $\left|h ; \bar{h}, \bar{j}^{+}, \bar{j}^{-}\right\rangle$. We find $\delta \tilde{c} \neq \delta c$ except for special cases such as the representations $\bar{j}^{+}=\bar{j}^{-}$or the limits $\gamma \rightarrow 0,1$. All entries should be multiplied by the spin-dependent factor $(-1)^{2(h-\bar{h})}$.

\begin{tabular}{|c|llll|}
\hline \multicolumn{1}{|c|}{ large } & \multicolumn{1}{l|}{} \\
$\mathcal{N}=(4,4)$ & long-long & long-short & short-long & short-short \\
\hline$E_{c}^{L}$ & 0 & 0 & 0 & $-\frac{1}{4}(1-2 \gamma)\left(j^{+}-j^{-}\right)$ \\
$E_{c}^{R}$ & 0 & 0 & 0 & $-\frac{1}{4}(1-2 \bar{\gamma})\left(\bar{j}^{+}-\bar{j}^{-}\right)$ \\
$\delta \tilde{c}$ & 0 & 0 & 0 & $3\left[(1-2 \gamma)\left(j^{+}-j^{-}\right)+(1-2 \bar{\gamma})\left(\bar{j}^{+}-\bar{j}^{-}\right)\right]$ \\
\hline$\delta c$ & 0 & 0 & 0 & $3\left[(1-2 \gamma)\left(j^{+}-j^{-}\right)+(1-2 \bar{\gamma})\left(\bar{j}^{+}-\bar{j}^{-}\right)\right]$ \\
\hline
\end{tabular}

Table 6. The left and right Casimir energies, $E_{c}^{L}$ and $E_{c}^{R}$, would-be central charge $\delta \tilde{c}$ and central charge $\delta c$ for large $\mathcal{N}=(4,4)$ multiplets. All multiplets have $\delta \tilde{c}=\delta c$. All entries should be multiplied by the spin-dependent factor $(-1)^{2(h-\bar{h})}$.

\subsubsection{Large $\mathcal{N}=(4,4)$}

Finally, we examine the large $\mathcal{N}=(4,4)$ case, or more precisely representations of $D(2,1 \mid \alpha) \times D(2,1 \mid \bar{\alpha})$. Again, we tensor together either long or short representations on the left and the right. The results summarized in table 6 show that in this case $\delta \tilde{c}=\delta c$.

Although we allow for different parameters on the left and the right in the discussion here, the case we have in mind is string theory on $\operatorname{AdS}_{3} \times S^{3} \times S^{3} \times S^{1}$ which has $\alpha=$ $\bar{\alpha}$, equivalent to $\gamma=\bar{\gamma}$. Furthermore, the BPS spectrum of Type II supergravity on $\mathrm{AdS}_{3} \times S^{3} \times S^{3} \times S^{1}$ only contains states with $j^{+}=j^{-}$and $\bar{j}^{+}=\bar{j}^{-}[24,25]$. In this special case all states in the Kaluza-Klein reduction therefore give vanishing contributions to $E_{c}^{L}, E_{c}^{R}$ and $\delta c$.

\subsection{How much supersymmetry is sufficient?}

As we have seen, the holographic dual to the CFT relation $\delta E_{c}=-\delta c / 12$ (equivalent to $\delta \tilde{c}=\delta c$ in the notation used in this section) is not generally upheld on a state by state basis in the bulk. In particular, it fails for bulk states with no supersymmetry at all and it similarly fails for $\mathcal{N}=(0,2)$ multiplets.

On the other hand, it holds for all theories with four or more supercharges, with the exception of large $\mathcal{N}=(0,4)$ supersymmetry where restriction to representations with $\bar{j}^{+}=\bar{j}^{-}$is needed. The restriction needed in the latter case may or may not be significant; it appears to be satisfied for all known models with large $\mathcal{N}=4$ supersymmetry in the bulk. 
This indicates that the standard Casimir energy may not be a useful tool for computing subleading central charges in $\mathrm{AdS}_{3} / \mathrm{CFT}_{2}$ duals with too little supersymmetry.

Of course, even in theories with fewer supercharges, we still expect $\delta E_{c}=-\delta c / 12$ to be valid once we sum over the complete bulk spectrum. However, the mechanism for this equality is far from obvious in the cases where it does not follow from supersymmetry applied to individual multiplets.

\section{Supersymmetric Casimir energy in $\mathrm{AdS}_{3}$}

In this section we propose an improved method for computing quantum corrections to the bulk central charge in $\mathrm{AdS}_{3} / \mathrm{CFT}_{2}$ holography. The linchpin is a supersymmetric version of the Casimir energy due to bulk fluctuations. This supersymmetric Casimir energy arises naturally in the context of partition functions that preserve some amount of supersymmetry which are closely related to the superconformal index.

As we will see, in the context of chiral $\mathcal{N}=(0,2)$ or $\mathcal{N}=(0,4)$ theories this approach will provide a useful tool in computing the left-moving subleading central charge $\delta c_{L}$. In theories where both chiralities preserve two or more supercharges we are able to compute quantum corrections to both the left and right-moving central charges in a manifestly supersymmetric fashion.

\subsection{The superconformal index and the supersymmetric Casimir energy}

In 2D CFTs with $\mathcal{N}=(0,2)$ supersymmetry the superconformal index is defined as

$$
\mathcal{I}_{R}(q)=\operatorname{Tr}(-1)^{2\left(L_{0}-\bar{L}_{0}\right)} q^{L_{0}} \bar{q}^{\bar{L}_{0}-\bar{J}_{0}}
$$

where the trace is over all states in the theory on the cylinder $S^{1} \times \mathbb{R}$. The index only receives contributions from states satisfying $\bar{L}_{0}=\bar{J}_{0}$. These only appear in shortened (chiral) multiplets with respect to a particular supercharge in the superconformal algebra. The index is thus independent of $\bar{q}$ and depends only on the BPS spectrum of the theory. We use a subscript $R$ to emphasize that the index localizes on multiplets that are shortened with respect to one of the right moving supercharges.

One can similarly define an anti-chiral index that localizes on states that are shortened with respect to the other supercharge. Also, in theories with a left-moving supersymmetry one can straightforwardly define analogous left-handed indices that localize onto shortened states of the left-moving algebra.

The index also has an interpretation as the partition function given by the Euclidean path-integral for the theory on $S^{1} \times S^{1}$, with fermions satisfying periodic boundary conditions around the temporal circle. The trace and the partition function representations of the index are related by an overall factor

$$
Z_{R}(q)=e^{-\beta E_{S U S Y}^{L}} \mathcal{I}_{R}(q) .
$$

Here $q=e^{-\beta}$, where $\beta$ is defined by the ratio of the length of the Euclidean time circle to the length of the spatial circle. We will often refer to $\beta$ as an inverse temperature even though 
we have given fermions periodic boundary conditions. The quantity $E_{S U S Y}^{L}$ in the exponent of the prefactor is the supersymmetric Casimir energy. We use a superscript $L$ in order to emphasize that in free field theory it is essentially a sum of eigenvalues of the left-moving $L_{0}$ over all the states killed by the right-moving supercharge (cf. eq. (4.12) below). Assuming a unique ground state and an appropriate gap above it so that the index satisfies $\mathcal{I}_{R}(q=$ $0)=1$, then $E_{S U S Y}^{L}$ can be extracted from the $\beta \rightarrow \infty$ limit of the partition function.

The supersymmetric Casimir energy has a universal structure determined by the anomaly polynomial of the SCFT. In particular, the Casimir energy determined from the right-handed partition function through (4.2) is given simply by the left-moving central charge [16]

$$
E_{\mathrm{SUSY}}^{L}=-\frac{c_{L}}{24}
$$

In theories that admit a holographic dual at large $c$ the central charge decomposes into a sum of a leading contribution derived from the classical supergravity action and a subleading contribution that is due to one-loop determinants of fields in the bulk. Similarly, the supersymmetric Casimir energy can be decomposed as

$$
E_{S U S Y}^{L}=E_{(0)}^{L}+E_{(1)}^{L} .
$$

The first term $E_{(0)}^{L}$ is a classical contribution from the bulk on-shell action evaluated on the $A d S_{3}$ background with an appropriate supersymmetric regularization prescription [26, 27]. ${ }^{4}$ The second term $E_{(1)}^{L}$ arises from one-loop contributions to the supersymmetric bulk partition function. In notation that is a natural generalization of the previous section one then has the relations

$$
E_{(0)}^{L}=-\frac{c_{L}^{(0)}}{24}
$$

and

$$
E_{(1)}^{L}=-\frac{\delta c_{L}}{24} .
$$

Our focus is on the computation of $E_{(1)}^{L}$ and will use (4.6) to relate it to $\delta c_{L}$.

In order to extract $\delta c_{L}$ we will utilize a particular property of the index at large $N$. Rewriting (4.2) as

$$
\mathcal{I}_{R}(q)=e^{\beta E_{S U S Y}^{L}} Z_{R}(q),
$$

and evaluating $Z_{R}(q)$ holographically for large $c$ limit we see that, since the large- $c$ index does not scale with $c$, the leading behavior at large $c$ arising from $E_{(0)}^{L}$ should cancel against the corresponding contribution to the partition function at large $c$. We can then simplify this to ${ }^{5}$

$$
\mathcal{I}_{R}^{\text {large- } c}(q)=e^{\beta E_{(1)}^{L}} Z_{R}^{\text {one-loop }}(q),
$$

\footnotetext{
${ }^{4}$ While references $[26,27]$ only consider the cases of $\mathrm{AdS}_{4}$ and $\mathrm{AdS}_{5}$, we expect that similar results also hold for $\mathrm{AdS}_{3}$.

${ }^{5}$ Our estimates all refer to the leading saddle point at large $c$ and quantum fluctuations around it. Additional contributions from subleading saddles can be neglected here because their suppression is of order $e^{-c}$.
} 
where $\mathcal{I}_{R}^{\text {large- } c}(q)$ is the $c \rightarrow \infty$ limit of the index and $Z_{R}^{\text {one-loop }}(q)$ refers to the contribution to the partition function from one-loop determinants of all bulk fields. At one-loop, the contributions to $Z_{R}(q)$ correspond to free fields in the bulk which have an interpretation as generalized free fields in the CFT.

In a high-temperature (small- $\beta$ ) expansion, the logarithm of the partition function for free fields has no term linear in $\beta$ [28-30]. Because of its generalized free field interpretation, we expect that $\ln Z_{R}^{\text {one-loop }}$ similarly has no term linear in $\beta$ in its small- $\beta$ expansion. We can therefore compute the quantum correction $E_{(1)}^{L}$ to the supersymmetric Casimir energy from the index at large $c$ and then infer the corresponding quantum correction $\delta c_{L}$ from (4.6). We substantiate this procedure in appendix $\mathrm{B}$, by demonstrating that the linear-in- $\beta$ term of $\ln \mathcal{I}_{R}^{\text {large-c }}$ can be reduced to a sum over left-moving energies of free single-particle states (annihilated by the right-handed supercharge), and so can naturally be identified with $E_{(1)}^{L}$ in the bulk.

\subsection{The single-particle index}

In theories with a holographic description the index at large $c$ is naturally expressed in terms of contributions from single-trace operators in the theory. For theories with $\mathcal{N}=(0,2)$ we express it as

$$
\mathcal{I}_{R}^{\text {s.t. }}(q)=\operatorname{Tr}_{\text {s.t. }}(-1)^{2\left(L_{0}-\bar{L}_{0}\right)} q^{L_{0}} \bar{q}^{\bar{L}_{0}-\bar{J}_{0}} .
$$

Each single-trace operator in the CFT is dual to a supergravity field, so in the bulk the trace can be thought of as being over single-particle states and we can replace "s.t." above with "s.p." While we will not use it in the following, we note for completeness that the full index (4.1) at large $c$ is constructed from the single-particle result by taking the plethystic exponential

$$
\mathcal{I}_{R}^{\text {large-c }}(q)=\exp \left(\sum_{k=1}^{\infty} \frac{1}{k} \mathcal{I}_{R}^{s . p .}\left(q^{k}\right)\right),
$$

which takes the multi-particle contributions into account .

The supersymmetric Casimir energy can be computed from the index in the same way that the standard Casimir energy was extracted from the partition function in (3.11), viz.

$$
E_{(1)}^{L}=-\left.\frac{1}{2} \lim _{\beta \rightarrow 0} \frac{d}{d \beta} \mathcal{I}_{R}^{s . p .}\left(q=e^{-\beta}\right)\right|^{\text {finite }} .
$$

We show in appendix B that this relation is equivalent to computing

$$
E_{(1)}^{L}=\sum(-1)^{2 s} \frac{1}{2} h_{n}
$$

using zeta-function regularization. It therefore has a natural interpretation as a Casimir energy (of the supersymmetric states) in the bulk, as expected.

Combining the supersymmetric Casimir energy (4.11) with (4.6) we have our main result

$$
\delta c_{L}=\left.12 \lim _{\beta \rightarrow 0} \frac{d}{d \beta} \mathcal{I}_{R}^{s . p .}\left(q=e^{-\beta}\right)\right|^{\text {finite }} .
$$


This provides an algorithm for computing the quantum corrections to the left-moving central charge in $\mathcal{N}=(0,2)$ supersymmetric theories with a holographic dual.

To find explicit formulae, we organize bulk states into multiplets of the $\mathcal{N}=(0,2)$ algebra, which come in two types: short and long. Short chiral multiplets satisfy $\bar{L}_{0}=\bar{J}_{0}$ and contribute to the index as

$$
\mathcal{I}_{R, h, \bar{h}}^{s . p .}(q)=(-1)^{2(h-\bar{h})} \frac{q^{h}}{1-q},
$$

where $h$ and $\bar{h}$ are the left and right-moving weights of the operator and the denominator arises from descendant contributions. Importantly, short anti-chiral multiplets (with $\bar{L}_{0}=$ $-\bar{J}_{0}$ ) and long multiplets (with $\bar{L}_{0}>\left|\bar{J}_{0}\right|$ ) give vanishing contributions to (4.9).

Inserting the single-particle index (4.14) for a chiral $\mathcal{N}=(0,2)$ multiplet into (4.11), we find

$$
E_{(1)}^{L}(h, \bar{h})=-\frac{1}{24}(-1)^{2(h-\bar{h})}\left(1-6 h+6 h^{2}\right),
$$

which gives

$$
\delta c_{L}(h, \bar{h})=(-1)^{2(h-\bar{h})}\left(1-6 h+6 h^{2}\right) .
$$

While for $\mathcal{N}=(0,2)$ theories we do not yet have a direct way of corroborating this result, when we apply it and its corresponding right-moving counterpart in theories with both left and right-moving supercharges we can compare with the Weyl anomaly results for $\delta c$. As we will see, the result in (4.16) corresponds to the sum of contributions to $\delta c_{L}$ from a chiral and the corresponding $\mathrm{CP}$ conjugate anti-chiral multiplet.

\subsection{Quantum corrections from one-loop Chern-Simons levels}

In an effort to support the veracity of our formula (4.16) for the central charge $\delta c_{L}$ in theories with only $\mathcal{N}=(0,2)$ supersymmetry it is useful to compute the right-moving central charge $\delta c_{R}$. This necessarily involves another method.

The central charge is related to the level $k_{R}$ of the right-moving $\mathrm{U}(1)_{R}$ current by

$$
\delta c_{R}=-3 \delta k_{R},
$$

where $\delta k_{R}$ is the bulk one-loop contribution to coefficient of the Chern-Simons term

$$
\delta \mathcal{L} \propto \frac{1}{4 \pi} \delta k_{R} A_{R} \wedge d A_{R}
$$

Holographic anomaly matching gives the $F_{R} \wedge F_{R}$ term in the $\mathrm{U}(1)_{R}$ anomaly. The quantum correction $\delta k_{R}$ can be computed from one-loop vacuum polarization diagrams in threedimensional flat space, extracting the contribution to the Chern-Simons term in the bulk. The topological nature of the Chern-Simons term implies that the flat space result is valid also in curved space. Explicit computations presented in appendix $\mathrm{C}$ give ${ }^{6}$

$$
\delta k_{R}(s, \bar{r})=-(-1)^{2 s} 2 s \bar{r}^{2},
$$

\footnotetext{
${ }^{6}$ We derive these results for spins $s=\frac{1}{2}, 1$ and $\frac{3}{2}$. While we do not consider spins greater than $\frac{3}{2}$, the simple extrapolation used here gives results consistent with expectations. It would be interesting to verify it by an explicit calculation. See also [32] for an independent computation of these Chern-Simons levels using an index theorem.
} 
for a field of spin $s=h-\bar{h}$ and right-moving $\mathrm{U}(1)_{R}$-charge $\bar{r}$. For an analogous calculation of one-loop Chern-Simons terms in five dimensions, see [31].

Summing the result (4.19) over the states in an $\mathcal{N}=(0,2)$ multiplet we find

$$
\begin{aligned}
\delta k_{R}^{\text {short }}(h, \bar{h}) & =-\frac{1}{4}(-1)^{2(h-\bar{h})}\left(1-4 \bar{h}^{2}-2 h-2 \bar{h}+8 h \bar{h}\right), \\
\delta k_{R}^{\text {long }}(h, \bar{h}) & =-\frac{1}{2}(-1)^{2(h-\bar{h})}(1-2 h+2 \bar{h}) .
\end{aligned}
$$

and then (4.17) gives the corresponding values for $\delta c_{R}$. Combining these results with our formula for $\delta c_{L}$, taking the degeneracies arising from chiral and anti-chiral multiplets into account, we arrive at values for the total central charge

$$
\delta c=\frac{1}{2}\left(\delta c_{L}+\delta c_{R}\right),
$$

for both short and long multiplets. The values for the Weyl anomaly $\delta c$ obtained this way agree with the results in table 1 , summed over a chiral multiplet and its CP conjugate antichiral multiplet. Importantly, we see also that $(0,2)$ supersymmetry is enough to ensure that long supermultiplets in the bulk do not contribute to $\delta c_{L}$. These observations provide evidence supporting our formula (4.11) computing $E_{(1)}^{L}$ from the right-handed index.

However, note that there is no a priori obvious way to regularize a sum of Chern-Simons levels over a Kaluza-Klein tower (without recourse to the higher-dimensional embedding as in [32]) as in the case of quantities computed directly from the index, such as $\delta c_{L}$. Therefore, utilizing the results in (4.20) and (4.21) to compute such a KK sum should be done with care.

\subsection{Higher amounts of supersymmetry}

In the remainder of this section we present results for cases with higher amounts of supersymmetry. We will see that the case of $\mathcal{N}=(0,4)$ is similar to $\mathcal{N}=(0,2)$ discussed above and that the story simplifies when there is both a left-moving and a right-moving supersymmetry.

\subsubsection{Small $\mathcal{N}=(0,4)$}

In the case of $\mathcal{N}=(0,4)$ supersymmetry, the index of a short multiplet is given by the same expression (4.14) as in the $\mathcal{N}=(0,2)$ case. This means that the result for $\delta c_{L}$ in (4.16) is also valid for short multiplets with $\mathcal{N}=(0,4)$ supersymmetry. Indeed, it matches exactly with the result from $E_{c}^{L}$ in table 3, upon identifying $E_{c}^{L}$ in section 3 with the $E_{(1)}^{L}$ defined in this section. This indicates that, for theories with at least four "small" supercharges, the somewhat ad hoc prescription pursued in section 3 is equivalent to the more principled method advanced in the current section, at least at the level of individual multiplets.

Note that for $\mathcal{N}=(0,4)$ we do not need to sum the results from table 3 over chiral and anti-chiral sectors as we did in the $\mathcal{N}=(0,2)$ case. This is because a short multiplet of $\mathcal{N}=(0,4)$ contains an entire $\mathrm{SU}(2)$ multiplet as its highest weight state. In particular, for each state in the highest weight representation the corresponding $\mathrm{CP}$ conjugate state is also included and one does not need to add the two together as in the case with $\mathcal{N}=(0,2)$. 
Like in the $\mathcal{N}=(0,2)$ case, we can check that our result for $\delta c_{L}$ is consistent with the Weyl anomaly $\delta c$ found in section 3 and presented in table 3 . For this comparison we compute the Chern-Simons levels for $(0,4)$ multiplets and find

$$
\begin{aligned}
\delta k_{R}^{\text {short }}(h, \bar{h}) & =(-1)^{2(h-\bar{h})} \bar{h}(1-2 h), \\
\delta k_{R}^{\text {long }}(h, \bar{h}) & =0,
\end{aligned}
$$

with the corresponding central charges again $\delta c_{R}=-3 \delta k_{R}$. Just as $\delta c_{L}$ found here was consistent with $E_{c}^{L}$ in table 3 , the result for $\delta k_{R}^{\text {short }}$ is consistent with $E_{c}^{R}$ in that table. With $\mathcal{N}=(0,4)$ supersymmetry the long multiplets offer no further check because, unlike the $\mathcal{N}=(0,2)$ case, the contribution from long multiplets to $\delta k_{R}$ vanishes.

\subsubsection{Large $\mathcal{N}=(0,4)$}

With large $\mathcal{N}=(0,4)$ supersymmetry, the index of a short multiplet is again given by the same expression (4.14) as in the $\mathcal{N}=(0,2)$ case, and hence the result for $\delta c_{L}$ in (4.16) is also valid for short multiplets with large $\mathcal{N}=(0,4)$ supersymmetry.

Interestingly, this result does not match with the one found using the ordinary Casimir energy $E_{c}^{L}$ and presented in table 5 , except for representations with $\bar{j}^{+}=\bar{j}^{-}$(or $\left.\gamma=0,1\right)$. Moreover, the discrepancies occur precisely in the situations where we had already identified problems with the $\mathrm{CFT}_{2}$ relation $\delta E_{c}=-\delta c / 12$. It is hard to escape the conclusion that the principled method using supersymmetric Casimir energy and a relation to an index is correct, while the prescription in section 3 is unreliable.

In this situation it is particularly important that we can check that the result for $\delta c_{L}$ is consistent with the quantum correction $\delta c$ to the holographic Weyl anomaly given in table 5, by performing an independent computation in Chern-Simons theory. The result for the Chern-Simons levels of the $\mathrm{U}(1)_{R}$ reported in (A.12) are

$$
\begin{aligned}
\delta k_{R}^{\text {short }}\left(h, \bar{h}, \bar{j}_{+}, \bar{j}_{-}\right) & =(-1)^{2(h-\bar{h})}\left(\bar{j}_{-}-\bar{j}_{+}\right)\left((1-2 \gamma)(1-2 h)-2 \gamma(1-\gamma)\left(\bar{j}_{+}-\bar{j}_{-}\right)\right), \\
\delta k_{R}^{\text {long }}\left(h, \bar{h}, \bar{j}_{+}, \bar{j}_{-}\right) & =0
\end{aligned}
$$

and again corresponds to central charge $\delta c_{R}=-3 \delta k_{R}$. We find agreement with the central charge $\delta c$ but generally not with the (presumably erroneous) result $\delta \tilde{c}$ that was deduced from the ordinary Casimir energy $E_{c}^{L}$.

As in the small $\mathcal{N}=(0,4)$ case, with large $\mathcal{N}=(0,4)$ the contribution from long multiplets to $\delta k_{R}$ vanishes. ${ }^{7}$

\subsection{3 $\mathcal{N}=(2,2)$}

When there is supersymmetry on both the left and right we can utilize the existence of a right-moving index $\mathcal{I}_{R}$ as well as a left-moving index $\mathcal{I}_{L}$ to compute both $\delta c_{L}$ and $\delta c_{R}$.

\footnotetext{
${ }^{7}$ This computation proceeds most easily if one first computes the sum over the $\mathrm{SU}(2) \times \mathrm{SU}(2)$ states in a representation of $\mathrm{SL}(2, R) \times \mathrm{SU}(2)^{2}$, giving

$$
\sum_{\left(\bar{j}^{+}, \bar{j}^{-}\right)} \bar{r}^{2}=\frac{1}{3}\left(1+2 \bar{j}^{+}\right)\left(1+2 \bar{j}^{-}\right)\left[\gamma^{2} \bar{j}^{+}\left(1+\bar{j}^{+}\right)+(1-\gamma)^{2} \bar{j}^{-}\left(1+\bar{j}^{-}\right)\right] .
$$
}




\begin{tabular}{|c|llll|}
\hline $\mathcal{N}=(2,2)$ & long-long & long-short & short-long & short-short \\
\hline$\delta c_{L}$ & 0 & 3 & 0 & $\frac{3}{2}(1-4 h)$ \\
$\delta c_{R}$ & 0 & 0 & 3 & $\frac{3}{2}(1-4 \bar{h})$ \\
\hline$\delta c$ & 0 & $\frac{3}{2}$ & $\frac{3}{2}$ & $\frac{3}{2}(1-2 h-2 \bar{h})$ \\
\hline
\end{tabular}

Table 7. The left and right central charges $\delta c_{L}$ and $\delta c_{R}$ as derived from the SUSY Casimir energy and the central charge $\delta c$ for $\mathcal{N}=(2,2)$ multiplets. The line $\delta c$ agrees with a sum of the results from table 2 over a chiral plus its CP conjugate anti-chiral multiplet. All entries should be multiplied by the spin-dependent factor $(-1)^{2(h-\bar{h})}$.

\begin{tabular}{|c|c|c|c|c|}
\hline \multicolumn{5}{|l|}{ small } \\
\hline $\mathcal{N}=(4,4)$ & long-long & long-short & short-long & short-short \\
\hline$\delta c_{L}$ & 0 & 0 & 0 & $-12 h$ \\
\hline$\delta c_{R}$ & 0 & 0 & 0 & $-12 \bar{h}$ \\
\hline$\delta c$ & 0 & 0 & 0 & $-6(h+\bar{h})$ \\
\hline
\end{tabular}

Table 8. The left and right central charges $\delta c_{L}$ and $\delta c_{R}$ as derived from the SUSY Casimir energy and the central charge $\delta c$ for $\mathcal{N}=(4,4)$ multiplets. The line $\delta c$ agrees with the result from table 4 . All entries should be multiplied by the spin-dependent factor $(-1)^{2(h-\bar{h})}$.

\begin{tabular}{|c|llll|}
\hline \multicolumn{2}{|c|}{ large } & & & \\
$\mathcal{N}=(4,4)$ & long-long & long-short & short-long & short-short \\
\hline$\delta c_{L}$ & 0 & 0 & 0 & $6\left[(1-2 \gamma)\left(j^{+}-j^{-}\right)\right]$ \\
$\delta c_{R}$ & 0 & 0 & 0 & $6\left[(1-2 \bar{\gamma})\left(\bar{j}^{+}-\bar{j}^{-}\right)\right]$ \\
\hline$\delta c$ & 0 & 0 & 0 & $3\left[(1-2 \gamma)\left(j^{+}-j^{-}\right)+(1-2 \bar{\gamma})\left(\bar{j}^{+}-\bar{j}^{-}\right)\right]$ \\
\hline
\end{tabular}

Table 9. The left and right central charges $\delta c_{L}$ and $\delta c_{R}$ as derived from the SUSY Casimir energy and the central charge $\delta c$ for large $\mathcal{N}=(4,4)$ multiplets. The line $\delta c$ corresponds to the result from table 6 . All entries should be multiplied by the spin-dependent factor $(-1)^{2(h-\bar{h})}$.

The results for $\delta c_{L}$ and $\delta c_{R}$ are presented in table 7. We find independent agreements with the chiral Casimir energies $E_{c}^{L}$ and $E_{c}^{R}$ reported in table 2, after summing the latter over a chiral multiplet plus its CP conjugate anti-chiral multiplet. This implies agreement also for the holographic Weyl anomaly

\subsubsection{Small and large $\mathcal{N}=(4,4)$}

With four supercharges on both the left and the right, we only find non-zero contributions to $\delta c_{L}$ and $\delta c_{R}$ from short-short multiplets. The results for the small $\mathcal{N}=(4,4)$ are summarized in table 8 and those for the large $\mathcal{N}=(4,4)$ are similarly given in table 9 . Both are completely consistent with the results from section 3, reported in table 4 and table 6 , respectively. 


\section{Quantum corrected central charge for specific dualities}

In this section we apply our formula for the quantum correction to the bulk central charge to full-fledged $\mathrm{AdS}_{3} / \mathrm{CFT}_{2}$ dualities in string theory. In these examples the $\mathrm{AdS}_{3}$ matter content is qualitatively more complicated than the individual multiplets considered in the previous sections. We must consider infinite KK towers, corresponding to supergravity fields in higher dimensions. The resulting divergences present challenges that are addressed correctly by our formula, at least in the examples we consider.

We first study cases where the full central charge $c$ is known from the boundary description; the challenge would then be to reproduce the $O(1)$ term in the central charge, denoted $\delta c$, from the bulk description.

In the last two subsections we move on to less-understood dualities where we will make predictions for the $O(1)$ piece of the central charges of the yet-to-be-discovered finite- $c$ boundary CFTs.

\section{$5.1 \quad \mathrm{AdS}_{3} \times S^{3} \times K 3$}

Type IIB theory on $\mathrm{AdS}_{3} \times S^{3} \times K 3$ has small $\mathcal{N}=(4,4)$ supersymmetry. The dual CFT is a $(K 3)^{Q_{1} Q_{5}+1} / S\left(Q_{1} Q_{5}+1\right)$ superconformal sigma model [33]. The seed theory with target space $K 3$ has central charge $c_{L}=c_{R}=6$ and the full symmetric orbifold has central charge $c_{L}=c_{R}=6\left(Q_{1} Q_{5}+1\right)$. The expansion parameter around the classical saddle point is $\frac{G_{\mathrm{AdS}_{3}}}{\ell_{\mathrm{AdS}_{3}}} \propto 1 / Q_{1} Q_{5}$ [34]. The $\mathrm{CFT}_{2}$ therefore has quantum corrections $\delta c_{L}=\delta c_{R}=6$.

The input to the $\mathrm{AdS}_{3}$ computation of $\delta c$ is the supersymmetric index. The KK towers comprising the supergravity spectrum was computed by de Boer $[35,36]$ who presented it in various forms including the one-particle index ${ }^{8}$

$$
\mathcal{I}_{R}^{\text {s.p. }}(q)=\mathcal{I}_{L}^{\text {s.p. }}(q)=1+\frac{44 \sqrt{q}+28 q}{1-q} .
$$

Applying the master formula (1.1) and its left-moving analogue we arrive at

$$
\delta c_{L}=\delta c_{R}=-\frac{864}{\beta^{2}}+6+O(\beta) \quad(\text { as } \beta \rightarrow 0) .
$$

The divergence in the $\beta \rightarrow 0$ limit is due to the infinite sum over towers of KaluzaKlein modes. Regularizing the divergence by expanding around $\beta=0$ and keeping the finite piece we obtain the desired result $\delta c_{L}=\delta c_{R}=6$. Our regularization is manifestly supersymmetric and similar to the one employed successfully in $\mathrm{AdS}_{5} / \mathrm{CFT}_{4}$ [7, 9]. The divergence could also be tamed more traditionally via a zeta-function regularization [10].

\section{$5.2 \quad \mathrm{AdS}_{3} \times S^{3} \times T^{4}$}

This case is also a duality with small $\mathcal{N}=(4,4)$ supersymmetry.

The $\mathrm{CFT}_{2}$ description is a $\sigma$-model on the target space $\tilde{T}^{4} \times\left(T^{4}\right)^{Q_{1} Q_{5}} / S\left(Q_{1} Q_{5}\right)$. The decoupled $\tilde{T}^{4}$ in the target space corresponds to the quantum Higgs branch of the D1D5 gauge theory [37]. From the viewpoint of the $\mathrm{U}\left(Q_{1}\right)$ gauge theory which flows to the

\footnotetext{
${ }^{8}$ This is the function $s(1, q, 1)$ in eq. (5.10) of [36] except that, since we are considering the "one"-particle index, we do not include the +1 vacuum contribution included in the reference.
} 
sigma model in the IR (see e.g. [38]), the $\tilde{T}^{4}$ represents an $\mathcal{N}=(4,4)$ hypermultiplet decoupled from the adjoint representation (see e.g. section 4 of [39]). The $\tilde{T}^{4}$ corresponds in the gravity picture to two pairs of $(1 / 2,0)_{s}+(0,1 / 2)_{s}$ singletons living on the boundary of $\mathrm{AdS}_{3}$. These two singletons are equivalent to the $\tilde{T}^{4}$ sigma model on the boundary. Therefore, as far as the $\tilde{T}^{4}$ factor is concerned, bulk and boundary theory are identical and their central charges match trivially: $c_{R}=c_{L}=6 .^{9}$

The more interesting part of the $\mathrm{CFT}_{2}$ is the $\left(T^{4}\right)^{Q_{1} Q_{5}} / S\left(Q_{1} Q_{5}\right)$ orbifold. The central charges are $c_{L}=c_{R}=6 Q_{1} Q_{5}$. Since the expansion parameter again is $\frac{G_{\mathrm{AdS}_{3}}}{\ell_{\mathrm{AdS}_{3}}} \propto 1 / Q_{1} Q_{5}[34]$ the central charges of the $\mathrm{CFT}_{2}$ are not corrected by quantum effects $\delta c_{L}=\delta c_{R}=0$.

The supersymmetric index for the excitations in the $\mathrm{AdS}_{3}$ is largely trivial, due to the high symmetry of $T^{4}$. Once the $(1 / 2,0)_{s}+(0,1 / 2)_{s}$ singletons are removed from the bulk spectrum the indices turn out to be ${ }^{10}$

$$
\mathcal{I}_{R}^{\text {s.p. }}(q)=\mathcal{I}_{L}^{\text {s.p. }}(q)=-1
$$

The prescription (1.1) thus gives the bulk quantum correction $\delta c_{L}=\delta c_{R}=0$, in agreement with the boundary dual.

Collecting the two parts of the analysis we find the quantum corrections $\delta c_{L}=\delta c_{R}=6$ for both bulk and boundary theory, with the nontrivial contributions from singletons and the overall $\tilde{T}^{4}$, respectively. BMT achieved this agreement in [10] using different methods.

\section{$5.3 \quad \mathrm{AdS}_{3} \times S^{3} \times S^{3} \times S^{1}$}

This case is a large $\mathcal{N}=(4,4)$ duality. The dual CFT is the $Q_{1} Q_{5}^{+}$-fold symmetric orbifold of a 2D CFT seed theory referred to as the $\mathcal{S}_{\kappa}$ theory [41] (and also [42, 43] for earlier works). For comparison with other references note that we take $\kappa \equiv \frac{Q_{5}^{-}}{Q_{5}^{+}}-1$. The central charges of this $\mathrm{CFT}_{2}$ are given by [41]

$$
c_{L}=c_{R}=\frac{6 Q_{1} Q_{5}^{-} Q_{5}^{+}}{Q_{5}^{-}+Q_{5}^{+}} .
$$

In the semi-classical gravity regime, the D-brane charges $Q_{1}$ and $Q_{5}^{+}$, as well as the D5-flux $Q_{5}^{-}$, are simultaneously taken to infinity as $Q_{1} \propto Q_{5}^{+} \propto Q_{5}^{-} \rightarrow \infty$. Moreover, there is a constraint that $\frac{Q_{5}^{-}}{Q_{5}^{+}} \in \mathbb{Z}^{>1}$. However, the expansion parameter is $\frac{G_{\mathrm{AdS}_{3}}}{\ell_{\mathrm{AdS}_{3}}} \propto 1 / Q_{1} Q_{5}^{+}[42]$ so the leading quantum correction vanishes $\delta c_{L}=\delta c_{R}=0$.

\footnotetext{
${ }^{9}$ This situation is analogous to the one-loop Weyl anomaly matching for the $\mathrm{U}(N) \mathcal{N}=4$ SYM and its bulk dual containing a "doubleton" living on the $\mathrm{AdS}_{5}$ boundary. Once the Weyl anomaly matching of the decoupled $\mathrm{U}(1)$ of the boundary and the doubleton of the bulk is established (essentially through their equivalence), it remains to show that the $\mathrm{SU}(N) \mathcal{N}=4 \mathrm{SYM}$ and the bulk theory without the doubleton have identical Weyl anomaly.

${ }^{10}$ Some authors add $\mathrm{a}+1$ vacuum contribution to this index, so it vanishes (cf. [40]). We avoid that in order to consider a "one"-particle index. The plethystic exponentiation of (5.3) yields a vanishing multiparticle index, as expected.
} 
In order to compute $\delta c_{L, R}$ from the bulk we start from the BPS spectrum of KK supergravity in this case $[24,25]$

$$
\bigoplus_{j \in \frac{1}{2} \mathbb{Z} \geq 0}^{\infty}\left([j, j]_{s}+\left[j+\frac{1}{2}, j+\frac{1}{2}\right]_{s}\right) \otimes\left([j, j]_{s}+\left[j+\frac{1}{2}, j+\frac{1}{2}\right]_{s}\right), .
$$

The notation $\left[j^{+}, j^{-}\right]_{s}$ indicates a short $_{j^{+}, j^{-}}$representation of $D(2,1 \mid \alpha)$. See the appendix for the details of the multiplet structure.

It is straightforward to check that each KK level $j$ in the spectrum (5.5) gives a vanishing contribution to the supergravity one-particle index. Since the $j=0$ level includes also the vacuum state whose contribution to the index we should not add, the total supergravity single-particle index comes out

$$
\mathcal{I}_{R}^{\text {s.p. }}(q)=\mathcal{I}_{L}^{\text {s.p. }}(q)=-1 \text {. }
$$

Then (1.1) establishes the $0=0$ match, very similarly to the $\mathcal{N}=(4,4)$ duality of the $\left(T^{4}\right)^{Q_{1} Q_{5}} / S\left(Q_{1} Q_{5}\right)$ sigma-model.

In this case, instead of going through the index, we could observe that both on the left and on the right, the short multiplets have $j^{+}=j^{-}$. However, subsection 3.3.6 established that such short $\times$ short multiplets do not contribute to $\delta c_{L, R}$. The $0=0$ agreement between quantum corrections in $\mathrm{CFT}_{2}$ and in $\mathrm{AdS}_{3}$ therefore follows effortlessly.

\subsection{M-theory on $\mathrm{AdS}_{3} \times S^{2} \times \mathrm{CY}_{3}$}

These compactifications are interesting because they describe large classes of 4D black holes [44]. Moreover, the duality has $\mathcal{N}=(0,4)$ supersymmetry and is the only chiral example we study in this paper.

The boundary CFT is a sigma model with left-moving central charge [44]

$$
c_{L}=P^{3}+c_{2} \cdot P-3,
$$

where $P$ is a very ample divisor in $C Y_{3}$ and $P^{3}$ is the triple self-intersection number of the divisor. We have subtracted the +3 contribution of the three translational zero-modes of the underlying M5-brane from the result of [44]. This subtraction is analogous to removing the decoupled $\tilde{T}^{4}$ from the boundary target space in the duality of subsection 5.2.

Because the expansion parameter is $\frac{G_{\mathrm{AdS}_{3}}}{\ell_{\mathrm{AdS}_{3}}} \propto 1 / P^{3}[45]$, we have $\delta c_{L}=-3$. The term that is linear in $P$ is due to higher-derivative corrections in bulk [19], rather then one-loop effects; so it is not our concern in this work.

Since we have supersymmetry on the right, in the bulk we can compute the righthanded index of the supergravity theory, and extract $\delta c_{L}$ from the index. To do so, we need the single-particle index of the KK supergravity. It is given in eq. (6.12) of [46]) as

$$
\mathcal{I}_{R}^{\text {s.p. }}(q)=2\left(h^{1,1}-h^{1,2}\right) \frac{q}{(1-q)^{2}}-3 \frac{q}{1-q},
$$

where $h^{i, j}$ are the hodge numbers of the $C Y_{3}$. Applying our master formula (1.1) we get

$$
\delta c_{L}=\frac{-48\left(h^{1,1}-h^{1,2}\right)}{\beta^{3}}+\frac{36}{\beta^{2}}-3
$$

with the finite piece (as $\beta \rightarrow 0$ ) exactly reproducing the expected boundary result.

Replacing the $C Y_{3}$ with $T^{6}$ or $K 3 \times T^{2}$ gives more subtle examples of $\mathrm{AdS}_{3} / \mathrm{CFT}_{2}$. We leave the holographic study of quantum corrections in these examples to the future. 


\section{5 $\quad \operatorname{AdS}_{3} \times\left(S^{3} \times T^{4}\right) / G$ and $\mathrm{AdS}_{3} \times\left(S^{3} \times K 3\right) / \mathbb{Z}_{2}$}

These cases are proposed $\mathcal{N}=(2,2)$ dualities [47]. The dual CFTs are not yet known at finite central charge. Therefore instead of presenting matchings, in this section we will be making predictions.

There are eight dualities in this class: seven dualities involving orbifolds of $S^{3} \times T^{4}$, and one duality involving a $\mathbb{Z}_{2}$ orbifold of $S^{3} \times K 3$. In all the eight cases the orbifold group $G$ rotates the $S^{3}$ such that a great circle $S^{1}$ remains fixed, but the action on the $M_{4}$ (i.e. $T^{4}$ or $\left.K 3\right)$ is free so that the internal geometry $\left(S^{3} \times M_{4}\right) / G$ is smooth. In the first seven cases $T^{4} / G$ is a Hyperelliptic Surface (HS), while in the eighth case $K 3 / \mathbb{Z}_{2}$ is the Enriques surface (ES). The proposed infinite- $c$ dual CFTs are infinite-fold symmetric product orbifolds with seed target spaces HS or ES, respectively.

For the seven HS cases the bulk one-particle indices are ${ }^{11}$

$$
\mathcal{I}_{R}^{\text {s.p. }}(q)=\mathcal{I}_{L}^{\text {s.p. }}(q)=-1,
$$

just like the case of $\mathrm{AdS}_{3} \times S^{3} \times T^{4}$ without the extra singletons, or the case of $\mathrm{AdS}_{3} \times$ $S^{3} \times S^{3} \times S^{1}$. Hence our prescription (1.1) gives the quantum correction

$$
\delta c_{L}^{H S}=\delta c_{R}^{H S}=0
$$

from the bulk side. Of course, this is disregarding possible bulk singletons that would be dual to decoupled factors in the boundary sigma model.

For the ES case, the bulk one-particle indices are nontrivial

$$
\mathcal{I}_{R}^{\text {s.p. }}(q)=\mathcal{I}_{L}^{\text {s.p. }}(q)=\frac{22 \sqrt{q}+14 q}{1-q} .
$$

where again we removed a +1 vacuum contribution from the result reported in [47]. Applying our master formula (1.1) we get

$$
\delta c_{L}^{E S}=\delta c_{R}^{E S}=-\frac{432}{\beta^{2}}+3,
$$

with the finite part (as $\beta \rightarrow 0$ ) giving our prediction for the boundary theory: $\delta c_{L}^{E S}=$ $\delta c_{R}^{E S}=3$.

\section{$5.6 \quad \operatorname{AdS}_{3} \times\left(S^{3} \times T^{4}\right) / D_{n}$}

As in the previous subsection, these cases are proposed $\mathcal{N}=(2,2)$ dualities [48]. The dual CFTs are not yet known at finite central charge, so in this subsection we will again be making predictions rather than presenting agreements.

There are a total of eight distinct cases. In the notation of [48], there are two for the $D_{1}$ group (the $D_{1}^{(1)}$ and $D_{1}^{(2)}$ cases), and six more for $D_{2}^{(1)}, D_{2}^{(2)}, D_{3}^{(1)}, D_{3}^{(2)}, D_{4}$, and $D_{6}$. The internal geometry $\left(S^{3} \times T^{4}\right) / D_{n}$ is singular in all the cases, so the bulk spectrum is not just KK modes from supergravity in higher dimensions, it also has a part coming from

\footnotetext{
${ }^{11}$ Reference [47] reports zero because it adds a +1 contribution from the vacuum.
} 
the twisted sector of IIB string theory. The proposed infinite- $c$ dual CFTs are infinite-fold symmetric product orbifolds with seed target space $T^{4} / D_{n}$.

For the two dualities with $D_{1}$ group, the bulk one-particle indices are simply -1 , just like the case of $\mathrm{AdS}_{3} \times S^{3} \times T^{4}$ without the extra singletons, the case of $\mathrm{AdS}_{3} \times S^{3} \times S^{3} \times S^{1}$, or the seven HS examples. Therefore, just as in those cases (1.1) gives

$$
\delta c_{L}^{D_{1}}=\delta c_{R}^{D_{1}}=0
$$

for the quantum correction computed from the bulk side. Of course, this is assuming no extra bulk singletons that would be dual to possible decoupled factors in the boundary sigma model.

For the remaining six dualities the superconformal index for single particle excitations in the bulk are nontrivial. It is given by eq. (6.33) of [41] as

$$
\mathcal{I}_{R}^{\text {s.p. }}(q)=\frac{4(1+a) q^{1 / 4}+22 \sqrt{q}+4(1+a) q^{3 / 4}+(18+4 a) q}{1-q},
$$

after setting the parameter in that work $z$ to zero, and again removing the +1 vacuum contribution included there. The parameter $a$ takes different values $\{1,-1 / 2,-1,-1,1 / 4,-1 / 2\}$ for the six distinct bulk geometries that we are considering. Our prescription (1.1) for the quantum correction to the central charge gives

$$
\delta c_{L}=\delta c_{R}=-\frac{144(4+a)}{\beta^{2}}+6\left(1+\frac{a}{2}\right)
$$

The finite piece, evaluated for the appropriate values of $a$, gives our predictions

$$
\delta c_{L}=\delta c_{R}=6(1+a / 2)=\{9,9 / 2,3,3,27 / 4,9 / 2\} .
$$

for the six boundary theories that are based on orbifold groups $D_{2}^{(1)}, D_{2}^{(2)}, D_{3}^{(1)}, D_{3}^{(2)}, D_{4}$, $D_{6}$.

\section{Summary and outlook}

The main result (1.1) of this article is an efficient prescription for the holographic computation of quantum corrections to central charges in the supersymmetric $\mathrm{AdS}_{3} / \mathrm{CFT}_{2}$ correspondence. Our one-loop (i.e. $\mathcal{O}\left(G_{\mathrm{AdS}_{3}}^{0}\right)$ ) formula follows from the relation between the central charges and the supersymmetric Casimir energies, as extracted from the hightemperature behavior of the bulk one-particle superconformal indices.

For theories with non-chiral supersymmetry where the left- and right-moving central charges are equal, our results agree with those of BMT [10], at the level of individual bulk multiplets. However, when considering complete Kaluza-Klein towers of states our method is advantageous because our regularization of the infinite KK sums is completely unambiguous and manifestly supersymmetric.

We also leveraged the notion of supersymmetric Casimir energy to motivate our formula even in cases with chiral supersymmetry. With "small" $\mathcal{N}=(0,4)$ SUSY our results 
again coincide with those of BMT [10] at the level of individual bulk multiplets, but not necessarily for entire KK towers. For multiplets of $\mathcal{N}=(0,2)$ or even multiplets of "large" $\mathcal{N}=(0,4)$ (for representations with $\bar{j}^{+} \neq \bar{j}^{-}$) we find disagreement.

Although it is possible that agreement would be restored after summing over the whole bulk spectrum, it is not obvious that this can be realized without a supersymmetric prescription for the sum. It would therefore be interesting to test our formula (1.1) for the KK spectra in instances of $\mathcal{N}=(0,2)$ and $\mathcal{N}=(0,4) \mathrm{AdS}_{3} / \mathrm{CFT}_{2}$, such as those in [49-51].

Among the concrete outcomes of the present work were the predictions in subsection 5.5 for the quantum corrections to central charges in the dualities recently discovered by Eberhardt [47]. Initial investigations seem to confirm our predictions, and reveal novel aspects of $\mathrm{AdS}_{3} / \mathrm{CFT}_{2}$ at the quantum level [52].

A more ambitious question left for future work is whether a version of our main result (1.1) can be found that applies at finite- $N$. Such a formula would relate the exact (left-handed) central charge of any 2D SCFT to its full (right-handed) superconformal index. A simple finite- $N$ version would be

$$
c_{L} \stackrel{?}{=} 12 \lim _{\beta \rightarrow 0} \frac{\mathrm{d}}{\mathrm{d} \beta} \operatorname{PL}\left(\mathcal{I}^{R}\left(q=e^{-\beta}\right)\right),
$$

where PL refers to the plethystic logarithm and $\mathcal{I}^{R}$ is the full right-handed SCFT index. A simple calculation shows that this proposal implies that the term that is linear in $\beta$ in the asymptotic small- $\beta$ expansion of $\ln \mathcal{I}^{R}$ is equal to $-\beta c_{L} / 24$.

Similar finite- $N$ prescriptions were conjectured for $4 \mathrm{~d}$ SCFTs in [9], but were later found to be invalid for SO(3) SQCD with two flavors [53]. This example shows that such finite- $N$ formulas do not necessarily apply when the Rains function of the $4 \mathrm{~d}$ SCFT has flat directions [53]. Such flat directions appear in turn related to unlifted Coulomb branches in the crossed channel - i.e. on $R^{3} \times S^{1}$ [53].

Analogously, we expect that (6.1) may be violated when certain gap conditions on the spectrum of the 2D SCFT are not met. With appropriate gap conditions, on the other hand, one can establish the proposal (6.1). For example, for $\mathcal{N}=(2,2)$ SCFTs with a gap in the spectrum above $L_{0}=J_{0}$ and with finite degeneracies (so that the "low-temperature" asymptotics of the index is dominated by the contribution of the states with $L_{0}=J_{0}$ ), it is a simple exercise to show that the modular properties of the elliptic genus imply (6.1). It is an interesting prospect that, ultimately, such gap conditions might have connections with the sparsity criteria (such as $[54,55]$ ) for a 2D SCFT having a holographic dual in the first place.

\section{Acknowledgments}

We would like to thank M. Beccaria, F. Benini, L. Eberhardt, H. het Lam, K. Mayer, D. McGady, S. Murthy, O. Sax, and S. Vandoren for helpful discussions and correspondences related to this project. The work of A.A.A. is supported by the Knut and Alice Wallenberg Foundation under grant Dnr KAW 2015.0083. The work of P.S. is part of the Delta ITP consortium, a program of the NWO that is funded by the Dutch Ministry of Education, Culture and Science (OCW). This work was supported in part also by the U.S. Department of Energy under grant DE-SC0007859. 


\section{A Some 2D superconformal representation theory}

In this appendix, we present a brief overview of superconformal representations used in the body of the paper. Our starting point is the $\mathcal{N}=2$ superconformal algebra, which can be further extended to yield the small and large $\mathcal{N}=4$ algebras. The two-dimensional conformal algebra decomposes into left- and right-moving sectors, and for the most part we will focus on a single copy. However, we also report the contribution of individual representations to the right-handed superconformal index

$$
\mathcal{I}_{R}(q)=\operatorname{Tr}(-1)^{2 L_{0}-2 \bar{L}_{0}} q^{L_{0}} \bar{q}^{\bar{L}_{0}-\bar{J}_{0}}
$$

When doing so, we will consider theories with equal amounts of supersymmetry on both sides. Note that this index assumes at least $\mathcal{N}=(0,2)$ supersymmetry, with $L_{0}$ and $J_{0}$ defined by the $\mathcal{N}=2$ algebra given below. The contributions to $\tilde{\mathcal{I}}_{R}(q)=$ $\operatorname{Tr}(-1)^{2 L_{0}-2 \bar{L}_{0}} q^{L_{0}} \bar{q}^{\bar{L}_{0}+\bar{J}_{0}}$ and the left-handed indices (assuming supersymmetry on the left) are obtained similarly.

\section{A.1 The $\mathcal{N}=2$ superconformal algebra}

The $\mathcal{N}=2$ superconformal algebra corresponds to $\mathrm{SU}(1,1 \mid 1)$, and it has a bosonic subgroup $\mathrm{SU}(1,1) \times \mathrm{U}(1)$. The global part of the algebra is given by $L_{0}, L_{ \pm 1}, G_{ \pm 1 / 2}^{+}, G_{ \pm 1 / 2}^{-}, J_{0}$, with corresponding (anti-)commutation relations

$$
\begin{aligned}
{\left[L_{0}, L_{ \pm 1}\right] } & =\mp L_{ \pm 1}, & {\left[L_{1}, L_{-1}\right] } & =2 L_{0}, \\
{\left[L_{0}, G_{ \pm 1 / 2}^{+}\right] } & =\mp \frac{1}{2} G_{ \pm 1 / 2}^{+}, & {\left[L_{0}, G_{ \pm 1 / 2}^{-}\right] } & =\mp \frac{1}{2} G_{ \pm 1 / 2}^{-}, \\
{\left[L_{1}, G_{-1 / 2}^{ \pm}\right] } & =G_{1 / 2}^{ \pm}, & {\left[L_{-1}, G_{1 / 2}^{ \pm}\right] } & =-G_{-1 / 2}^{ \pm}, \\
\left\{G_{ \pm 1 / 2}^{+}, G_{ \pm 1 / 2}^{-}\right\} & =2 L_{ \pm 1}, & \left\{G_{ \pm 1 / 2}^{+}, G_{\mp 1 / 2}^{-}\right\} & =2\left(L_{0} \pm J_{0}\right), \\
{\left[J_{0}, G_{1 / 2}^{ \pm}\right] } & = \pm \frac{1}{2} G_{1 / 2}^{ \pm}, & {\left[J_{0}, G_{-1 / 2}^{ \pm}\right] } & = \pm \frac{1}{2} G_{-1 / 2}^{ \pm} .
\end{aligned}
$$

Lowest weight representations are built from the state $|h, r\rangle$ where $h$ and $r$ are the $L_{0}$ and $J_{0}$ eigenvalues, respectively. The full representation is then obtained by acting with a combination of the creation operators $L_{-1}, G_{-1 / 2}^{+}$and $G_{-1 / 2}^{-}$.

Representations of the $\mathcal{N}=2$ superconformal algebra. Unitary irreducible representations exist for $h \geq|r|$. The case $h>|r|$ gives rise to an $\mathcal{N}=2$ long representation built from four $\mathcal{N}=0$ representations

$|h, r\rangle,\left|h+\frac{1}{2}, r+\frac{1}{2}\right\rangle=G_{-1 / 2}^{+}|h, r\rangle,\left|h+\frac{1}{2}, r-\frac{1}{2}\right\rangle=G_{-1 / 2}^{-}|h, r\rangle,|h+1, r\rangle=G_{-1 / 2}^{+} G_{-1 / 2}^{-}|h, r\rangle$.

When $h=|r|$, we end up with a shortened representation. The chiral representation corresponds to starting with $|h, h\rangle$, in which case $G_{-1 / 2}^{+}$creates a null state. As a result, the chiral multiplet is generated by $L_{-1}$ and $G_{-1 / 2}^{-}$. The $\mathcal{N}=0$ decomposition is

$$
|h, h\rangle, \quad\left|h+\frac{1}{2}, h-\frac{1}{2}\right\rangle=G_{-1 / 2}^{-}|h, h\rangle .
$$


Finally, the antichiral multiplet is built from

$$
|h,-h\rangle, \quad\left|h+\frac{1}{2}, \frac{1}{2}-h\right\rangle=G_{-1 / 2}^{+}|h,-h\rangle .
$$

Contribution to the index. Focusing on the right-handed index (A.1), it is easy to see that long and antichiral representations on the right do not contribute. Hence only chiral multiplets on the right contribute, giving a factor $(-1)^{-2 \bar{h}}$ for the $|\bar{h}, \bar{h}\rangle$ multiplet. As a result, we have

$$
\mathcal{I}_{R}(q)\left[\text { any } \times \operatorname{chiral}_{\overline{\mathrm{h}}}\right]=\operatorname{Tr}_{L}(-1)^{2\left(L_{0}-\bar{h}\right)} q^{L_{0}},
$$

where the trace is taken over the left-moving representation. For $\mathcal{N}=(2,2)$ supersymmetry, the only non-vanishing contributions are then

$$
\begin{aligned}
& \mathcal{I}_{R}(q)\left[\operatorname{long}_{h, r} \times \operatorname{chiral}_{\bar{h}}\right]=(-1)^{2(h-\bar{h})} \frac{(1-\sqrt{q})^{2} q^{h}}{1-q}, \\
& \mathcal{I}_{R}(q)\left[\operatorname{short}_{h} \times \operatorname{chiral}_{\bar{h}}\right]=(-1)^{2(h-\bar{h})} \frac{(1-\sqrt{q}) q^{h}}{1-q},
\end{aligned}
$$

where the short representation on the left can be either chiral or antichiral. It is now straightforward to check that the prescription (1.1) acting on $\mathcal{I}_{R}(q)$ successfully reproduces the result of table 7 .

\section{A.2 The small $\mathcal{N}=4$ superconformal algebra}

The $\mathcal{N}=4$ superconformal algebra corresponds to $\operatorname{PSU}(1,1 \mid 2)$, with bosonic subgroup $\mathrm{SU}(1,1) \times \mathrm{SU}(2)$. The global part of the algebra is given by $L_{0}, L_{ \pm 1}, G_{ \pm 1 / 2}^{a}, \tilde{G}_{ \pm 1 / 2}^{a}$ and $J_{0}^{i}$. Lowest weight representations are built from the state $|h, j, m=j\rangle$, where $h$ is the $L_{0}$ eigenvalue and $j, m$ are the $\mathrm{SU}(2)$ quantum numbers. This state is annihilated by $L_{1}$, $G_{1 / 2}^{a}, \tilde{G}_{1 / 2}^{a}, J_{1}^{i}$ and $J_{0}^{+}$. Descendants are built using the creation operators $L_{-1}, G_{-1 / 2}^{a}$, $\tilde{G}_{-1 / 2}^{a}$ as well as the $\mathrm{SU}(2)$ generators $J_{0}^{i}$.

Representations of the small $\mathcal{N}=4$ superconformal algebra. Representations come in two forms, long (massive, with $h>j$ ) and short (massless, with $h=j$ ). The long representations are generated by acting with all four supercharge creation operators $G_{-1 / 2}^{a}$ and $\tilde{G}_{-1 / 2}^{a}$, transforming as $\mathbf{2}+\overline{\mathbf{2}}$ of $\mathrm{SU}(2)$. A generic long representation is built from 16 $\mathcal{N}=0$ representations, all transforming as representations of $\mathrm{SU}(2)$

$$
\begin{array}{lll}
|h, j\rangle, & \\
2\left|h+\frac{1}{2}, j+\frac{1}{2}\right\rangle, & 2\left|h+\frac{1}{2}, j-\frac{1}{2}\right\rangle, & \\
|h+1, j+1\rangle, & 4|h+1, j\rangle, & \\
2\left|h+\frac{3}{2}, j+\frac{1}{2}\right\rangle, & 2\left|h+\frac{3}{2}, j-\frac{1}{2}\right\rangle, & \\
|h+2, j\rangle . &
\end{array}
$$

These representations decompose into a number of long $\mathcal{N}=2$ multiplets, and hence do not contribute to the index. 
A short representation of the $\mathcal{N}=4$ superconformal algebra has $h=j$. Starting from $|j, j, m=j\rangle$, we can see that $G_{-1 / 2}^{2}$ and $\tilde{G}_{-1 / 2}^{1}$ generate null states. We thus use $G_{-1 / 2}^{1}$ and $\tilde{G}_{-1 / 2}^{2}$ to generate descendants. The short multiplet contains

$$
|j, j\rangle, \quad 2\left|j+\frac{1}{2}, j-\frac{1}{2}\right\rangle, \quad|j+1, j-1\rangle .
$$

In particular, these short representations decompose into one chiral, one antichiral, and a number of long $\mathcal{N}=2$ multiplets. Therefore if such an $\mathcal{N}=4$ short representation is present on the right sector, it contributes to $\mathcal{I}_{R}$ just as its constituent $\mathcal{N}=2$ chiral multiplet does, namely $(-1)^{-2 \bar{h}}$.

Contribution to the index. The right-handed index only receives contributions from shortened representations on the right

$$
\mathcal{I}_{R}(q)\left[\text { any } \times \operatorname{short}_{\bar{h}}\right]=\operatorname{Tr}_{L}(-1)^{2\left(L_{0}-\bar{h}\right)} q^{L_{0}} .
$$

For the non-chiral $\mathcal{N}=(4,4)$ algebra, we have

$$
\begin{aligned}
& \mathcal{I}_{R}(q)\left[\operatorname{long}_{h, r} \times \operatorname{short}_{\bar{h}}\right]=(-1)^{2(h-\bar{h})} \frac{(2 h+1)(1-\sqrt{q})^{3} q^{h}}{1+\sqrt{q}}, \\
& \mathcal{I}_{R}(q)\left[\operatorname{short}_{h} \times \operatorname{short}_{\bar{h}}\right]=(-1)^{2(h-\bar{h})} \frac{(1+\sqrt{q}+2 h(1-\sqrt{q})) q^{h}}{1+\sqrt{q}} .
\end{aligned}
$$

The short $_{h} \times$ short $_{\bar{h}}$ representations of the $\mathcal{N}=(4,4)$ algebra are sometimes denoted as $(h, \bar{h})_{s}$. It is straightforward to check that the prescription (1.1) acting on the above successfully reproduces the result of table 8 .

\section{A.3 The large $\mathcal{N}=4$ superconformal algebra}

The bulk supergravity symmetry group for the duality involving the large $\mathcal{N}=(4,4)$ SCA is $D(2,1 \mid \alpha) \times D(2,1 \mid \alpha)$. Therefore we focus our discussion on a single copy of $D(2,1 \mid \alpha)$. The bosonic subgroup of $D(2,1 \mid \alpha)$ is $\mathrm{SL}(2, R) \times \mathrm{SU}(2) \times \mathrm{SU}(2)$. Therefore three quantum numbers $h, j^{+}, j^{-}$will be used to label its lowest weight representations. Note that the large $\mathcal{N}=4$ algebra has an extra $\mathrm{U}(1)$, but only the singlet sector of that $\mathrm{U}(1)$ is of interest to us. Hence the corresponding quantum number never appears in our discussion.

For comparison with the better known $\mathcal{N}=2$ algebra, note that $D(2,1 \mid \alpha)$ has an $\mathcal{N}=2$ subgroup, whose $\operatorname{SL}(2, R)$ part coincides with that of $D(2,1 \mid \alpha)$, and whose $\mathrm{U}(1)$ part is given by the combination

$$
J^{\mathcal{N}=2}=\gamma J_{z}^{+}+(1-\gamma) J_{z}^{-}
$$

of the third-component generators of the two SU(2)'s inside $D(2,1 \mid \alpha)$, where $\gamma=\alpha /(1+\alpha)$.

Representations of $\boldsymbol{D}(\mathbf{2}, \mathbf{1} \mid \boldsymbol{\alpha})$. Unitary representations of $D(2,1 \mid \alpha)$ satisfy the BPS inequality

$$
h \geq \gamma j^{+}+(1-\gamma) j^{-}
$$




$\left|h, j^{+}, j^{-}\right\rangle$,
$\left|h+\frac{1}{2}, j^{+}+\frac{1}{2}, j^{-}+\frac{1}{2}\right\rangle, \quad\left|h+\frac{1}{2}, j^{+}+\frac{1}{2}, j^{-}-\frac{1}{2}\right\rangle, \quad\left|h+\frac{1}{2}, j^{+}-\frac{1}{2}, j^{-}+\frac{1}{2}\right\rangle, \quad\left|h+\frac{1}{2}, j^{+}-\frac{1}{2}, j^{-}-\frac{1}{2}\right\rangle$,
$\left|h+1, j^{+}+1, j^{-}\right\rangle,\left|h+1, j^{+}, j^{-}+1\right\rangle, 2\left|h+1, j^{+}, j^{-}\right\rangle,\left|h+1, j^{+}-1, j^{-}\right\rangle,\left|h+1, j^{+}, j^{-}-1\right\rangle$,
$\left|h+\frac{3}{2}, j^{+}+\frac{1}{2}, j^{-}+\frac{1}{2}\right\rangle, \quad\left|h+\frac{3}{2}, j^{+}+\frac{1}{2}, j^{-}-\frac{1}{2}\right\rangle, \quad\left|h+\frac{3}{2} j^{+}-\frac{1}{2}, j^{-}+\frac{1}{2}\right\rangle, \quad\left|h+\frac{3}{2}, j^{+}-\frac{1}{2}, j^{-}-\frac{1}{2}\right\rangle$,
$\left|h+2, j^{+}, j^{-}\right\rangle$.

Table 10. The long representations of $D(2,1 \mid \alpha)$, where $h>\gamma j^{+}+(1-\gamma) j^{-}$.

\begin{tabular}{|lll}
$\left|h, j^{+}, j^{-}\right\rangle$, & \\
$\left|h+\frac{1}{2}, j^{+}+\frac{1}{2}, j^{-}-\frac{1}{2}\right\rangle$, & $\left|h+\frac{1}{2}, j^{+}-\frac{1}{2}, j^{-}+\frac{1}{2}\right\rangle$, & $\left|h+\frac{1}{2}, j^{+}-\frac{1}{2}, j^{-}-\frac{1}{2}\right\rangle$, \\
$\left|h+1, j^{+}, j^{-}\right\rangle$, & $\left|h+1, j^{+}-1, j^{-}\right\rangle$, & $\left|h+1, j^{+}, j^{-}-1\right\rangle$, \\
$\left|h+\frac{3}{2}, j^{+}-\frac{1}{2}, j^{-}-\frac{1}{2}\right\rangle$. & \\
\hline
\end{tabular}

Table 11. The short representations of $D(2,1 \mid \alpha)$, where $h=\gamma j^{+}+(1-\gamma) j^{-}$.

with short representations saturating the bound. The long representations of $D(2,1 \mid \alpha)$ are shown in table 10, and the short representations are shown in table 11. Note that only short representations with $j^{+}=j^{-}=j$ appear in the spectrum of string theory on $\mathrm{AdS}_{3} \times S^{3} \times S^{3} \times S^{1}[24,25]$. For such representations, the BPS relation becomes $h=j$.

Contribution to the index. Long representations of $D(2,1 \mid \alpha)$ can be decomposed into a number of long $\mathcal{N}=2$ multiplets, and hence do not contribute to the right-handed superconformal index. On the other hand, short representations of $D(2,1 \mid \alpha)$ can yield short $\mathcal{N}=2$ multiplets, and hence can contribute to the index. To see how this arises, we compare the $D(2,1 \mid \alpha)$ shortening condition, $h=\gamma j^{+}+(1-\gamma) j^{-}$, with the $\mathcal{N}=2$ shortening condition, $h=|r|$. Using the relation (A.12) between the $\mathcal{N}=2$ current and the $D(2,1 \mid \alpha)$ currents then demonstrates that a short $D(2,1 \mid \alpha)$ representation contains one chiral and one anti-chiral $\mathcal{N}=2$ multiplet, along with a set of long $\mathcal{N}=2$ multiplets. The chiral (anti-chiral) multiplet corresponds to the state within $\left|h, j^{+}, j^{-}\right\rangle$where the third components of the two SU(2)'s are both maximal (minimal).

As a result, the contribution of a short $D(2,1 \mid \alpha)$ multiplet on the right to $\mathcal{I}_{R}(q)$ takes the form

$$
\mathcal{I}_{R}(q)\left[\text { any } \times \operatorname{short}_{\bar{j}^{+}, \overline{\mathrm{j}}^{-}}\right]=\operatorname{Tr}_{L}(-1)^{2\left(L_{0}-\bar{h}\right)} q^{L_{0}} .
$$

The contribution of $D(2,1 \mid \alpha) \times D(2,1 \mid \alpha)$ representations to the right-handed index can then be determined to be

$$
\begin{aligned}
& \mathcal{I}_{R}(q)\left[\operatorname{long}_{h, j^{+}, j^{-}} \times \operatorname{short}_{j^{+}, j^{-}}\right]=\frac{(-1)^{2(h-\bar{h})}\left(2 j^{+}+1\right)\left(2 j^{-}+1\right)(1-\sqrt{q})^{4} q^{h}}{1-q}, \\
& \mathcal{I}_{R}(q)\left[\operatorname{short}_{j^{+}, j^{-}} \times \operatorname{short}_{j^{+}, \bar{j}^{-}}\right] \\
& =\frac{(-1)^{2(h-\bar{h})}\left(1+2\left(j^{+}+j^{-}\right)(1-\sqrt{q})+4 j^{+} j^{-}(1-\sqrt{q})^{2}+\sqrt{q}\right) q^{h}}{1+\sqrt{q}} .
\end{aligned}
$$

Once again, we can verify that (1.1) acting the index successfully reproduces the result of table 9 . 


\section{B Zeta function representation of the large- $c$ index and the supersym- metric Casimir energy}

In the main text we have focused on the relation between the supersymmetric Casimir energy and the single-particle supersymmetric index. The single-particle index is advantageous for practical computations but the full multi-particle index would perhaps be conceptually more satisfying. Happily, the relation between the two is particularly simple in the large- $c$ limit. In this appendix we show how the supersymmetric Casimir energy as given in (4.12) naturally appears as a linear-in- $\beta$ term in the natural logarithm of the full multi-particle large- $c$ index. Additionally, we show how this term is related to the standard definition of the Casimir energy in terms of a regulated sum over energy eigenvalues which appears as the zero temperature contribution to the free energy. We stress that we are not presenting a $\mathrm{CFT}_{2}$ discussion in this appendix, but studying the $\mathrm{AdS}_{3}$ side.

\section{B.1 Supersymmetric Casimir energy from the index}

Starting from the large- $c$ single-particle index in (4.9), the full index is given by enumerating all multi-particle states which arise as products of single-particle states. This is accomplished by taking the Plethystic exponential as in (4.10), which we reproduce here

$$
\mathcal{I}_{R}^{\text {large-c }}(q)=\exp \left(\sum_{k=1}^{\infty} \frac{1}{k} \mathcal{I}_{R}^{\text {s.p. }}\left(q^{k}\right)\right) .
$$

To simplify the notation we will omit the large-c superscript in the following.

For concreteness, consider an $\mathcal{N}=(0,2)$ chiral multiplet (cases with higher amounts of supersymmetry can be treated similarly). The single-particle index (4.14) of such a chiral multiplet is

$$
\mathcal{I}_{R, h, \bar{h}}^{s . p}(q)=(-1)^{2(h-\bar{h})} \frac{q^{h}}{1-q}=(-1)^{2(h-\bar{h})} \sum_{n=0}^{\infty} q^{h+n} .
$$

Inserting this into (B.1), the natural logarithm of the large- $c$ index is given by the series

$$
\ln \mathcal{I}_{R, h, \bar{h}}\left(e^{-\beta}\right)=(-1)^{2(h-\bar{h})} \sum_{k=1}^{\infty} \sum_{n=0}^{\infty} \frac{1}{k} e^{-k \beta(h+n)},
$$

where we have set $q=e^{-\beta}$.

In order to extract the small- $\beta$ asymptotics of this expression, we follow Cardy [56] and write the exponential as the inverse Mellin transform of the Gamma function

$$
e^{-x}=\frac{1}{2 \pi i} \int_{\mathcal{C}} x^{-s} \Gamma(s) d s,
$$

where the contour runs to the right of and parallel to the imaginary axis. Inserting this into the logarithm of the index we have

$$
\ln \mathcal{I}_{R, h, \bar{h}}\left(e^{-\beta}\right)=(-1)^{2(h-\bar{h})} \frac{1}{2 \pi i} \int_{\mathcal{C}} \beta^{-s} \zeta(s+1) \Gamma(s) \sum_{n=0}^{\infty}(h+n)^{-s} d s,
$$


where $\zeta(s+1)$ comes from the sum over $k$ in (B.3). The contour is now to the right of $\operatorname{Re} s=1$ where the integrand is convergent. This contour can be closed by deforming the upper $(\operatorname{Im} s>0)$ and lower $(\operatorname{Im} s<0)$ segments up and down towards the negative real axis from above and below. Cauchy's theorem then gives the small- $\beta$ expansion

$$
\ln \mathcal{I}_{R, h, \bar{h}}\left(e^{-\beta}\right)=(-1)^{2(h-\bar{h})}\left(\frac{1}{\beta} \frac{\pi^{2}}{6}+\zeta^{\prime}(h ; 0)+\beta \frac{1}{2} \zeta(h ;-1)+\cdots\right)
$$

where

$$
\zeta(h ; s)=\sum_{n=0}^{\infty}(h+n)^{-s},
$$

is a Hurwitz zeta function. The dots in (B.6) refer to higher powers in $\beta$ that arise from the infinite set of poles in the $\Gamma$-function at $s=-n$ for integer $n>1$. These terms are subleading for small $\beta$.

We are particularly interested in the term that is linear in $\beta$. It is due to the pole of the integrand in (B.5) at $s=-1$ which arises from the $\Gamma$-function. To find its coefficient we have used $\operatorname{Res}(\Gamma,-1)=-1$ as well as $\zeta(0)=-1 / 2 .{ }^{12}$ We now recognize that the coefficient of $\beta$ in (B.6) is nothing but the sum (4.12) over the left-handed quantum zero-energies of the supersymmetric $\mathrm{AdS}_{3}$ modes in the multiplet, and thus it gives $E_{(1)}^{L}(h, \bar{h})$ as claimed. Of course, the Hurwitz zeta function that appears in this term is elementary so we can evaluate the coefficient of $\beta$ in (B.6) explicitly and find

$$
\frac{1}{2}(-1)^{2(h-\bar{h})} \zeta(h ;-1)=-\frac{1}{24}(-1)^{2(h-\bar{h})}\left(1-6 h+6 h^{2}\right)
$$

This matches our result in (4.15) that was derived from the single-particle index using the prescription (4.11).

That the coefficient of $\beta$ in the small- $\beta$ expansion of $\ln \mathcal{I}_{R}$ should be $E_{(1)}^{L}$ follows from our prescription (4.11) as well; the reader might convince themselves of this using (B.1), or more carefully using the relation between the small- $\beta$ asymptotics of the single-particle and multi-particle indices presented in appendix D of [57].

To summarize this appendix so far, we have shown that

- the supersymmetric Casimir energy $E_{(1)}^{L}$ that we obtained from a single-particle-index regularization (4.11) of the expression (4.12), also arises as the coefficient of $\beta$ in the small- $\beta$ asymptotic expansion of logarithm of the bulk multi-particle index;

- at the level of individual bulk multiplets, the index prescription (4.11) and the zetafunction regularization (B.8) amount to the same result for the expression (4.12).

\footnotetext{
${ }^{12}$ To get the other terms shown in (B.6) we have used $\operatorname{Res}(\Gamma, 0)=1$ and $\zeta(2)=\pi^{2} / 6$ for the $\beta^{-1}$ term. For the $\beta^{0}$ term, we note that the Laurent expansion of $\zeta(s+1) \Gamma(s)=1 / s^{2}+\mathcal{O}\left(s^{0}\right)$ near $s=0$, which gives rise to the derivative $\left.\zeta^{\prime}(0 ; h) \equiv \frac{\partial}{\partial s} \zeta(s ; h)\right|_{s=0}$ appearing in the residue of the pole at this point.
} 


\section{B.2 Supersymmetric Casimir energy as a sum over normal mode energies}

We now want to show that the SUSY Casimir energy in (B.8) corresponds to a sum over supersymmetric energies of states in the bulk chiral multiplet.

To see this note that a field in global $\mathrm{AdS}_{3}$ with conformal weight $(h, \bar{h})$ has quantized normal mode energies [58]

$$
E_{n, l}(h, \bar{h})=\frac{2 n+|l|+h+\bar{h}}{\ell_{3}}, \quad n=0,1,2, \ldots, l=0, \pm 1, \pm 2, \ldots,
$$

where $l$ is the "angular" momentum around the spatial circle, $n$ is a radial mode number and $\ell_{3}$ is the AdS radius. We will set $\ell_{3}=1$ in the following.

In the supersymmetric context that we consider, these energies are modified due to the presence of a constant background $\mathrm{U}(1)_{R}$ gauge field that is required in order for the bulk partition function in global $\mathrm{AdS}_{3}$ to correspond to the supersymmetric partition function (or elliptic genus) [46]. ${ }^{13}$ This constant background $\mathrm{U}(1)_{R}$ gauge field is a flat connection so it simply shifts the energies by an amount equal to the $R$-charge such that the supersymmetric energies become

$$
E_{n, l}^{S U S Y}(h, \bar{h} ; j)=2 n+|l|+h+\bar{h}-\bar{r},
$$

where $n$ and $l$ have the same ranges as in (B.9) and $\bar{r}$ is the $\mathrm{U}(1)_{R}$ charge of the field.

Each entry of the oscillator spectrum (B.10) contributes the usual vacuum energy $\frac{1}{2} \hbar \omega$. The supersymmetric Casimir energy follows as a renormalized sum over these contributions so using zeta-function regularization we find:

$$
E^{S U S Y}(h, \bar{h} ; \bar{r}):=\left.\frac{1}{2}(-1)^{2(h-\bar{h})} \sum_{\kappa=0}^{\infty}(\kappa+1)(\kappa+h+\bar{h}-\bar{r})^{-s}\right|_{s=-1} .
$$

We have inserted an extra $(-1)^{2(h-\bar{h})}$ in order to count bosons with a plus sign and fermions with a minus sign. Also, in (B.11) we have replaced the double sum on $n$ and $l$ with a single sum that takes into account the appropriate degeneracy of each energy level.

For the $\mathcal{N}=(0,2)$ chiral multiplet in (A.4), the $\mathrm{U}(1)_{R}$ charge is related to the weight by $\bar{r}=\bar{h}$, so we have

$$
E^{c h}(h, \bar{h} ; \bar{r}=\bar{h})=\frac{1}{2}(-1)^{2(h-\bar{h})} \sum_{\kappa=0}^{\infty}\left[(\kappa+1)(\kappa+h)^{-s}-(\kappa+1)(\kappa+h+1)^{-s}\right]_{s=-1} .
$$

After shifting the summation index in the second term, this can be written

$$
E^{c h}(h, \bar{h})=\left.\frac{1}{2}(-1)^{2(h-\bar{h})} \sum_{\kappa=0}^{\infty}(\kappa+h)^{-s}\right|_{s=-1},
$$

which is equal to the SUSY Casimir energy defined from the index in (B.6).

On the other hand, one can check that for the long $\mathcal{N}=(0,2)$ multiplet in (A.3) the sum over the supersymmetric energies vanishes due to cancellation between bosons and fermions.

\footnotetext{
${ }^{13}$ See also $[26,27]$ for the corresponding statements in $\mathrm{AdS}_{5}$.
} 


\section{Computation of one-loop Chern-Simons terms}

In this appendix we collect some details of the computation of the Chern-Simons levels claimed in (4.18) of section 4, for spins less than two. It amounts to computing gauge boson vacuum polarization diagrams in flat space with charged fields running in the loop and extracting the parity-odd term that survives in the low-energy limit. We proceed directly to the vacuum polarization computation and only then providing the full context. The main result is given in equation (4.19), where the spin $s$ is given from the results below by identifying $\operatorname{sign}(m)=\frac{s}{|s|}[59]$.

The generation of Chern-Simons couplings in the three-dimensional effective action from one-loop vacuum polarization diagrams due to spin- $1 / 2$ fermions was first found in [60, 61 ], which we generalize to fields of spin- 1 and spin- $3 / 2$ and conjecture an extrapolation to higher spins. The analysis of this section follows the methods and regularization of [31] where the analogous calculation in five dimensions is performed.

\section{C.1 Details on the vacuum polarization diagrams}

The integrand of the one-loop diagrams we will consider are all of the form

$$
\Sigma^{\mu \nu}(p)=\int \frac{d^{3} k}{(2 \pi)^{3}} \frac{\mathbb{N}^{\mu \nu}(p, k ; m)}{\left(k^{2}+m^{2}\right)\left((k-p)^{2}+m^{2}\right)},
$$

where $p^{\mu}$ is the external photon momentum and $k^{\mu}$ is the loop momentum.

Here we will go through some of the details in extracting the relevant terms in the numerator factors. In particular, since we are interested in extracting the parity odd term we can restrict to the terms in the numerator of the form

$$
\mathbb{N}^{\mu \nu}(p, k ; m)=f\left(k^{2} ; m\right) \epsilon^{\mu \nu \rho} p_{\rho} .
$$

In particular, we can discard terms with higher powers of $p$ as well as those that do not include the Levi-Civita tensor.

\section{C.1.1 Spin-1/2}

For fermions, the relevant parity odd terms arise from traces of $\gamma$ matrices. The spin- $1 / 2$ propagator is

$$
\Delta^{1 / 2}(p)=\frac{-\not p+i m}{p^{2}+m^{2}}
$$

and the interaction vertex is simply

$$
V_{1 / 2}^{\mu}=q \gamma^{\mu}
$$

From these, we construct the numerator for a spin-1/2 field in the loop to be

$$
\begin{aligned}
\mathbb{N}_{1 / 2}^{\mu \nu}(p, k ; m) & =-\operatorname{Tr}\left[\left(-q \gamma^{\mu}\right)(-\not k+i m)\left(-q \gamma^{\nu}\right)(-(\not k-\not p)+m)\right] \\
& \simeq q^{2}\left[-\operatorname{Tr}\left(\gamma^{\mu} \gamma^{\rho} \gamma^{\nu}\right)\left(i m k_{\rho}\right)-\operatorname{Tr}\left(\gamma^{\mu} \gamma^{\nu} \gamma^{\rho}\right)\left(i m\left(k_{\rho}-p_{\rho}\right)\right)\right] \\
& \simeq 2 i m q^{2}\left[-\epsilon^{\mu \rho \nu} k_{\rho}-\epsilon^{\mu \nu \rho}\left(k_{\rho}-p_{\rho}\right)\right] \\
& \simeq 2 i m q^{2} \epsilon^{\mu \nu \rho} p_{\rho} .
\end{aligned}
$$


Putting this all together we find the following parity-odd contribution to the effective action

$$
\begin{aligned}
\sum_{1 / 2}^{\mu \nu} e_{\mu} e_{\nu} & =(-) \int \frac{d^{3} k}{(2 \pi)^{3}} \operatorname{Tr}\left(\left(-q \gamma^{\mu}\right) \frac{-\not k+i m}{k^{2}+m^{2}}\left(-q \gamma^{\nu}\right) \frac{-(\not k-\not p)+i m}{(k-p)^{2}+m^{2}}\right) \\
& \simeq-2 i m q^{2} p_{\rho} e_{\mu} e_{\nu} \epsilon^{\rho \mu \nu} \int \frac{d^{3} k}{(2 \pi)^{3}} \frac{1}{\left(k^{2}+m^{2}\right)\left((k-p)^{2}+m^{2}\right)} \\
& \simeq-\frac{i}{2 \pi^{2}} \frac{m}{|m|} q^{2} \Gamma\left(\frac{3}{2}\right) \Gamma\left(\frac{1}{2}, \Lambda^{-2}\right) p_{\rho} e_{\mu} e_{\nu} \epsilon^{\rho \mu \nu} \\
& \simeq \frac{i}{4 \pi} \operatorname{sign}(m) q^{2} p_{\rho} e_{\mu} e_{\nu} \epsilon^{\rho \mu \nu} .
\end{aligned}
$$

\section{C.1.2 Spin-1}

For bosons, the parity odd term arises from explicit $\epsilon$ tensors in both the propagator and the vertex. For spin-1, these are

$$
\begin{aligned}
\Delta_{\mu \nu}^{1}(p) & =-i\left(m \eta_{\mu \nu}+\frac{1}{m} p_{\mu} p_{\nu}-i \epsilon_{\mu \nu \gamma} p^{\gamma}\right), \\
V_{1}^{\nu \mu \lambda} & =-q \epsilon^{\nu \mu \lambda} .
\end{aligned}
$$

These give the numerator

$$
\begin{aligned}
\mathbb{N}_{1}^{\mu \nu}(p, k ; m) & =\left(-q \epsilon^{\lambda \mu \sigma}\right) \Delta_{\lambda \rho}^{1}(k)\left(-q \epsilon^{\rho \nu \gamma}\right) \Delta_{\gamma \sigma}^{1}(k-p), \\
& \simeq-i m q^{2} \epsilon^{\mu \nu \sigma} p_{\sigma}\left(1-\frac{k^{2}}{m^{2}}\right) .
\end{aligned}
$$

From which we compute

$$
\begin{aligned}
\Sigma_{1}^{\mu \nu} e_{\mu} e_{\nu} & =(+) \int \frac{d^{3} k}{(2 \pi)^{3}} \frac{\left(-q \epsilon^{\lambda \mu \sigma}\right) \Delta_{\lambda \rho}^{1}(k)\left(-q \epsilon^{\rho \nu \gamma}\right) \Delta_{\gamma \sigma}^{1}(k-p)}{\left(k^{2}+m^{2}\right)\left((k-p)^{2}+m^{2}\right)} \\
& \simeq-i m q^{2} p_{\rho} e_{\mu} e_{\nu} \epsilon^{\rho \mu \nu} \int \frac{d^{3} k}{(2 \pi)^{3}}\left(1-\frac{k^{2}}{m^{2}}\right) \frac{1}{\left(k^{2}+m^{2}\right)\left((k-p)^{2}+m^{2}\right)} \\
& \simeq-\frac{i}{4 \pi^{2}} \frac{m}{|m|} q^{2}\left(\Gamma\left(\frac{3}{2}\right) \Gamma\left(\frac{1}{2}, \Lambda^{-2}\right)-\Gamma\left(\frac{5}{2}\right) \Gamma\left(-\frac{1}{2}, \Lambda^{-2}\right)\right) p_{\rho} e_{\mu} e_{\nu} \epsilon^{\rho \mu \nu} \\
& \simeq \frac{i}{2 \pi}\left(\frac{3 \Lambda}{4 \sqrt{\pi}}-1\right) \operatorname{sign}(m) q^{2} p_{\rho} e_{\mu} e_{\nu} \epsilon^{\rho \mu \nu}
\end{aligned}
$$

\section{C.1.3 Spin-3/2}

For massive spin-3/2 fields in the loop, we have

$$
\begin{aligned}
\Delta_{\mu \nu}^{3 / 2}(p) & =\left(\eta_{\mu \nu}+\frac{p_{\mu} p_{\nu}}{m^{2}}\right)(-\not p+i m)+\frac{1}{2}\left(\gamma_{\mu}-\frac{i p_{\mu}}{m}\right)(-\not p-i m)\left(\gamma_{\nu}-\frac{i p_{\nu}}{m}\right), \\
V^{\nu \mu \lambda} & =-q \gamma^{\nu \mu \lambda} .
\end{aligned}
$$

These give a numerator of

$$
\mathbb{N}_{3 / 2}^{\mu \nu}(p, k ; m)=-\operatorname{Tr}\left[\left(-q \epsilon^{\lambda \mu \sigma}\right) \Delta_{\lambda \rho}^{\frac{3}{2}}(k)\left(-q \epsilon^{\rho \nu \gamma}\right) \Delta_{\gamma \sigma}^{\frac{3}{2}}(k-p)\right] .
$$


To simplify this, schematically write

$$
\Delta_{\mu \nu}^{\frac{3}{2}}(p)=A_{\mu \nu}(p)+B_{\mu \nu}(p)
$$

where $A_{\mu \nu}(p)$ refers to the first term in (C.10) and $B_{\mu \nu}(p)$ to the second. The numerator can then be written

$$
\mathbb{N}_{3 / 2}^{\mu \nu}(p, k ; m)=-q^{2} \epsilon^{\lambda \mu \sigma} \epsilon^{\rho \nu \gamma} \operatorname{Tr}\left[\left(A_{\lambda \rho}(k)+B_{\lambda \rho}(k)\right)\left(A_{\gamma \sigma}(k-p)+B_{\gamma \sigma}(k-p)\right)\right] .
$$

Evaluating the various terms in the product independently we find

$$
\begin{aligned}
\epsilon^{\lambda \mu \sigma} \epsilon^{\rho \nu \gamma} \operatorname{Tr}\left[A_{\lambda \rho}(k) A_{\gamma \sigma}(k-p)\right] & \simeq 0 \\
\epsilon^{\lambda \mu \sigma} \epsilon^{\rho \nu \gamma} \operatorname{Tr}\left[A_{\lambda \rho}(k) B_{\gamma \sigma}(k-p)\right] & \simeq-i m \epsilon^{\mu \nu \rho} p_{\rho}\left(1+\frac{k^{2}}{m^{2}}\right), \\
\epsilon^{\lambda \mu \sigma} \epsilon^{\rho \nu \gamma} \operatorname{Tr}\left[B_{\lambda \rho}(k) A_{\gamma \sigma}(k-p)\right] & \simeq-i m \epsilon^{\mu \nu \rho} p_{\rho}\left(1+3 \frac{k^{2}}{m^{2}}\right), \\
\epsilon^{\lambda \mu \sigma} \epsilon^{\rho \nu \gamma} \operatorname{Tr}\left[B_{\lambda \rho}(k) B_{\gamma \sigma}(k-p)\right] & \simeq 2 i m \epsilon^{\mu \nu \rho} p_{\rho}\left(1+\frac{k^{2}}{m^{2}}\right) .
\end{aligned}
$$

Adding everything together we find the numerator is given by

$$
\mathbb{N}_{3 / 2}^{\mu \nu}(p, k ; m)=-2 i m q^{2} k^{2} \epsilon^{\mu \nu \rho} p_{\rho} .
$$

Putting it altogether, we find the effective parity odd term

$$
\begin{aligned}
\sum_{3 / 2}^{\mu \nu} e_{\mu} e_{\nu} & =(-) \int \frac{d^{3} k}{(2 \pi)^{3}} \frac{\operatorname{Tr}\left[\left(-q \epsilon^{\delta \mu \rho}\right) \Delta_{\delta \sigma}^{\frac{3}{2}}(k)\left(-q \epsilon^{\lambda \nu \sigma}\right) \Delta_{\lambda \rho}^{\frac{3}{2}}(k-p)\right]}{\left(k^{2}+m^{2}\right)\left((k-p)^{2}+m^{2}\right)} \\
& \simeq-2 i m q^{2} p_{\rho} e_{\mu} e_{\nu} \epsilon^{\rho \mu \nu} \int \frac{d^{3} k}{(2 \pi)^{3}} \frac{k^{2}}{m^{2}} \frac{1}{\left(k^{2}+m^{2}\right)\left((k-p)^{2}+m^{2}\right)} \\
& \simeq-\frac{i}{2 \pi^{2}} \frac{m}{|m|} q^{2} \Gamma\left(\frac{5}{2}\right) \Gamma\left(-\frac{1}{2}, \Lambda^{-2}\right) p_{\rho} e_{\mu} e_{\nu} \epsilon^{\rho \mu \nu} \\
& \simeq-\frac{i}{2 \pi}\left(\frac{3 \Lambda}{2 \sqrt{\pi}}-\frac{3}{2}\right) \operatorname{sign}(m) q^{2} p_{\rho} e_{\mu} e_{\nu} \epsilon^{\rho \mu \nu} .
\end{aligned}
$$

\section{C.1.4 Extrapolation to arbitrary spin}

The results above all have a finite term given by

$$
\Sigma_{|s|}^{\mu \nu} e_{\mu} e_{\nu}=-(-1)^{2 s} \frac{i}{2 \pi} s q^{2} p_{\rho} e_{\mu} e_{\nu} \epsilon^{\rho \mu \nu},
$$

where $s$ is the spin of the field appearing in the loop, which is related to the mass by $m=\frac{s}{|s|}$. Given the chiral nature of the massive higher spin fields in three-dimensions [59], it is reasonable to suspect that charged massive higher spin fields will continue to follow this pattern. This suspicion is corroborated by the results in section 4 . It would be very interesting to verify this conjectured structure with an explicit calculation, but we will leave this for future work. 


\section{C.2 Momentum integrals}

In order to compute the momentum integrals we have utilized the Schwinger parametrization of the integrand. Here are some expressions relevant for this.

Since we are only interested in terms in the effective action of the form $p_{\rho} e_{\mu} e_{\nu} \epsilon^{\rho \mu \nu}$, we can restrict to the terms from the loop integrals which are independent of the external momentum $p$.

One can rewrite the denominator as

$$
\frac{1}{\left(k^{2}+m^{2}\right)\left((k-p)^{2}+m^{2}\right)}=\int_{0}^{\infty} d u \int_{0}^{\infty} d v e^{-(u+v)\left(\ell^{2}+\bar{m}^{2}\right)}
$$

where we have defined the shifted momentum $\ell$ and the shifted mass $\bar{m}^{2}$ as

$$
\begin{aligned}
\ell & =k-\frac{v}{u+v} p, \\
\bar{m}^{2} & =m^{2}+\frac{u v}{(u+v)^{2}} p^{2} .
\end{aligned}
$$

Since we are interested in the $p$-independent part of the integral, we drop the $p$ dependence of these.

The integral over the momentum can now be computed by evaluating the Gaussian integral

$$
\int \frac{d^{3} \ell}{(2 \pi)^{3}} \ell^{2 n} e^{-\frac{x \ell^{2}}{m^{2}}}=\frac{|m|^{2 n+3}}{4 \pi^{2}} \frac{\Gamma\left(n+\frac{3}{2}\right)}{(u+v)^{n+3 / 2}} .
$$

Putting this all together we have

$$
\begin{aligned}
\int \frac{d^{3} k}{(2 \pi)^{3}} \frac{k^{2 n}}{\left(k^{2}+m^{2}\right)\left((k-p)^{2}+m^{2}\right)} & =\frac{1}{2 \pi^{2}} \frac{m^{2 n}}{|m|} \Gamma\left(n+\frac{3}{2}\right) \int d u d v \Theta\left(u+v-\Lambda^{-2}\right) \frac{e^{-(u+v)}}{(u+v)^{n+3 / 2}} \\
& =\frac{1}{4 \pi^{2}} \frac{m^{2 n}}{|m|} \Gamma\left(n+\frac{3}{2}\right) \Gamma\left(-n+\frac{1}{2}, \Lambda^{-2}\right) .
\end{aligned}
$$

In the last line we used

$$
\int_{0}^{\infty} d u \int_{0}^{\infty} d v \Theta\left(u+v-\Lambda^{-2}\right) \frac{e^{-(u+v)}}{(u+v)^{\alpha}}=\Gamma\left(2-\alpha, \Lambda^{-2}\right)
$$

The parameter $\Lambda$ is inserted as a UV cut-off. In this way the Schwinger variables act as a heat kernel regulator of the loop integral and $\Lambda$ provides a UV cut-off for integration over the heat variables $u$ and $v$. One can then perform the integral and expand in large- $\Lambda$. The regularization procedure is to simply discard the infinite terms proportional to positive powers of $\Lambda$.

Open Access. This article is distributed under the terms of the Creative Commons Attribution License (CC-BY 4.0), which permits any use, distribution and reproduction in any medium, provided the original author(s) and source are credited. 


\section{References}

[1] M. Henningson and K. Skenderis, The holographic Weyl anomaly, JHEP 07 (1998) 023 [hep-th/9806087] [INSPIRE].

[2] A. Bilal and C.-S. Chu, A note on the chiral anomaly in the AdS/CFT correspondence and $1 / N^{2}$ correction, Nucl. Phys. B 562 (1999) 181 [hep-th/9907106] [INSPIRE].

[3] P. Mansfield and D. Nolland, Order $1 / N^{2}$ test of the Maldacena conjecture: cancellation of the one loop Weyl anomaly, Phys. Lett. B 495 (2000) 435 [hep-th/0005224] [INSPIRE].

[4] P. Mansfield, D. Nolland and T. Ueno, Order $1 / N^{2}$ test of the Maldacena conjecture 2. The full bulk one loop contribution to the boundary Weyl anomaly, Phys. Lett. B 565 (2003) 207 [hep-th/0208135] [INSPIRE].

[5] A. Arabi Ardehali, J.T. Liu and P. Szepietowski, The spectrum of IIB supergravity on $A d S_{5} \times S^{5} / Z_{3}$ and a $1 / N^{2}$ test of $A d S / C F T, J H E P 06$ (2013) 024 [arXiv:1304.1540] [INSPIRE].

[6] A. Arabi Ardehali, J.T. Liu and P. Szepietowski, $1 / N^{2}$ corrections to the holographic Weyl anomaly, JHEP 01 (2014) 002 [arXiv:1310.2611] [INSPIRE].

[7] A. Arabi Ardehali, J.T. Liu and P. Szepietowski, $c-a$ from the $N=1$ superconformal index, JHEP 12 (2014) 145 [arXiv: 1407.6024] [INSPIRE].

[8] M. Beccaria and A.A. Tseytlin, Higher spins in $A d S_{5}$ at one loop: vacuum energy, boundary conformal anomalies and AdS/CFT, JHEP 11 (2014) 114 [arXiv:1410.3273] [INSPIRE].

[9] A. Arabi Ardehali, J.T. Liu and P. Szepietowski, Central charges from the $N=1$ superconformal index, Phys. Rev. Lett. 114 (2015) 091603 [arXiv:1411.5028] [INSPIRE].

[10] M. Beccaria, G. Macorini and A.A. Tseytlin, Supergravity one-loop corrections on AdS $S_{7}$ and $A d S_{3}$, higher spins and AdS/CFT, Nucl. Phys. B 892 (2015) 211 [arXiv:1412.0489] [INSPIRE].

[11] H.-C. Kim and S. Kim, M5-branes from gauge theories on the 5-sphere, JHEP 05 (2013) 144 [arXiv:1206.6339] [INSPIRE].

[12] H.-C. Kim, S. Kim, S.-S. Kim and K. Lee, The general M5-brane superconformal index, arXiv:1307.7660 [INSPIRE].

[13] B. Assel, D. Cassani and D. Martelli, Localization on Hopf surfaces, JHEP 08 (2014) 123 [arXiv:1405.5144] [INSPIRE].

[14] A. Arabi Ardehali, J.T. Liu and P. Szepietowski, High-temperature expansion of supersymmetric partition functions, JHEP 07 (2015) 113 [arXiv:1502.07737] [INSPIRE].

[15] B. Assel, D. Cassani, L. Di Pietro, Z. Komargodski, J. Lorenzen and D. Martelli, The Casimir energy in curved space and its supersymmetric counterpart, JHEP 07 (2015) 043 [arXiv: 1503.05537] [INSPIRE].

[16] N. Bobev, M. Bullimore and H.-C. Kim, Supersymmetric Casimir energy and the anomaly polynomial, JHEP 09 (2015) 142 [arXiv: 1507.08553] [INSPIRE].

[17] H.W.J. Bloete, J.L. Cardy and M.P. Nightingale, Conformal invariance, the central charge and universal finite size amplitudes at criticality, Phys. Rev. Lett. 56 (1986) 742 [INSPIRE].

[18] I. Affleck, Universal term in the free energy at a critical point and the conformal anomaly, Phys. Rev. Lett. 56 (1986) 746 [InSPIRE].

[19] P. Kraus and F. Larsen, Microscopic black hole entropy in theories with higher derivatives, JHEP 09 (2005) 034 [hep-th/0506176] [INSPIRE]. 
[20] J.D. Brown and M. Henneaux, Central charges in the canonical realization of asymptotic symmetries: an example from three-dimensional gravity, Commun. Math. Phys. 104 (1986) 207 [INSPIRE].

[21] S. Giombi and I.R. Klebanov, One loop tests of higher spin AdS/CFT, JHEP 12 (2013) 068 [arXiv: 1308.2337] [INSPIRE].

[22] R.R. Metsaev, Massless mixed symmetry bosonic free fields in d-dimensional anti-de Sitter space-time, Phys. Lett. B 354 (1995) 78 [INSPIRE].

[23] J. de Boer, A. Pasquinucci and K. Skenderis, AdS/CFT dualities involving large $2 D N=4$ superconformal symmetry, Adv. Theor. Math. Phys. 3 (1999) 577 [hep-th/9904073] [INSPIRE].

[24] M. Baggio, O. Ohlsson Sax, A. Sfondrini, B. Stefański and A. Torrielli, Protected string spectrum in $A d S_{3} / C F T_{2}$ from worldsheet integrability, JHEP 04 (2017) 091 [arXiv: 1701.03501] [INSPIRE].

[25] L. Eberhardt, M.R. Gaberdiel, R. Gopakumar and W. Li, BPS spectrum on $A d S_{3} \times S^{3} \times S^{3} \times S^{1}$, JHEP 03 (2017) 124 [arXiv: 1701.03552] [INSPIRE].

[26] P. Benetti Genolini, D. Cassani, D. Martelli and J. Sparks, The holographic supersymmetric Casimir energy, Phys. Rev. D 95 (2017) 021902 [arXiv: 1606. 02724] [InSPIRE].

[27] P. Benetti Genolini, D. Cassani, D. Martelli and J. Sparks, Holographic renormalization and supersymmetry, JHEP 02 (2017) 132 [arXiv: 1612.06761] [INSPIRE].

[28] G. Plunien, B. Müller and W. Greiner, The Casimir effect, Phys. Rept. 134 (1986) 87 [INSPIRE].

[29] G. Plunien, B. Muller and W. Greiner, Casimir energy at finite temperature, Physica A 145 (1987) 202 [INSPIRE].

[30] G.W. Gibbons, M.J. Perry and C.N. Pope, Partition functions, the Bekenstein bound and temperature inversion in anti-de Sitter space and its conformal boundary, Phys. Rev. D 74 (2006) 084009 [hep-th/0606186] [INSPIRE].

[31] F. Bonetti, T.W. Grimm and S. Hohenegger, One-loop Chern-Simons terms in five dimensions, JHEP 07 (2013) 043 [arXiv:1302.2918] [INSPIRE].

[32] T.W. Grimm, H. het Lam, K. Mayer and S. Vandoren, Four-dimensional black hole entropy from F-theory, JHEP 01 (2019) 037 [arXiv: 1808.05228] [INSPIRE].

[33] C. Vafa, Instantons on D-branes, Nucl. Phys. B 463 (1996) 435 [hep-th/9512078] [INSPIRE].

[34] A. Strominger, Black hole entropy from near horizon microstates, JHEP 02 (1998) 009 [hep-th/9712251] [INSPIRE].

[35] J. de Boer, Six-dimensional supergravity on $S^{3} \times A d S_{3}$ and $2 D$ conformal field theory, Nucl. Phys. B 548 (1999) 139 [hep-th/9806104] [INSPIRE].

[36] J. de Boer, Large N elliptic genus and AdS/CFT correspondence, JHEP 05 (1999) 017 [hep-th/9812240] [INSPIRE].

[37] E. Witten, On the conformal field theory of the Higgs branch, JHEP 07 (1997) 003 [hep-th/9707093] [INSPIRE].

[38] O. Ohlsson Sax, A. Sfondrini and B. Stefanski, Integrability and the conformal field theory of the Higgs branch, JHEP 06 (2015) 103 [arXiv:1411.3676] [INSPIRE].

[39] J.R. David, G. Mandal and S.R. Wadia, Microscopic formulation of black holes in string theory, Phys. Rept. 369 (2002) 549 [hep-th/0203048] [INSPIRE]. 
[40] J.M. Maldacena, G.W. Moore and A. Strominger, Counting BPS black holes in toroidal type II string theory, hep-th/9903163 [INSPIRE].

[41] L. Eberhardt, M.R. Gaberdiel and W. Li, A holographic dual for string theory on $A d S_{3} \times S^{3} \times S^{3} \times S^{1}, J H E P 08(2017) 111$ [arXiv: 1707.02705] [INSPIRE].

[42] S. Gukov, E. Martinec, G.W. Moore and A. Strominger, The search for a holographic dual to $A d S_{3} \times S^{3} \times S^{3} \times S^{1}, A d v$. Theor. Math. Phys. 9 (2005) 435 [hep-th/0403090] [InSPIRE].

[43] D. Tong, The holographic dual of $A d S_{3} \times S^{3} \times S^{3} \times S^{1}$, JHEP 04 (2014) 193 [arXiv: 1402.5135] [INSPIRE].

[44] J.M. Maldacena, A. Strominger and E. Witten, Black hole entropy in M-theory, JHEP 12 (1997) 002 [hep-th/9711053] [INSPIRE].

[45] J.M. Maldacena, The large $N$ limit of superconformal field theories and supergravity, Int. J. Theor. Phys. 38 (1999) 1113 [hep-th/9711200] [INSPIRE].

[46] P. Kraus and F. Larsen, Partition functions and elliptic genera from supergravity, JHEP 01 (2007) 002 [hep-th/0607138] [INSPIRE].

[47] L. Eberhardt, Supersymmetric AdS 3 supergravity backgrounds and holography, JHEP 02 (2018) 087 [arXiv: 1710.09826] [INSPIRE].

[48] S. Datta, L. Eberhardt and M.R. Gaberdiel, Stringy $N=(2,2)$ holography for AdS $S_{3}$, JHEP 01 (2018) 146 [arXiv:1709.06393] [INSPIRE].

[49] B. Haghighat, S. Murthy, C. Vafa and S. Vandoren, F-theory, spinning black holes and multi-string branches, JHEP 01 (2016) 009 [arXiv: 1509.00455] [INSPIRE].

[50] C. Couzens, C. Lawrie, D. Martelli, S. Schäfer-Nameki and J.-M. Wong, F-theory and $A d S_{3} / C F T_{2}, J H E P 08$ (2017) 043 [arXiv: 1705.04679] [INSPIRE].

[51] C. Couzens, D. Martelli and S. Schäfer-Nameki, F-theory and $A d S_{3} / C F T_{2}(2,0), J H E P 06$ (2018) 008 [arXiv:1712.07631] [INSPIRE].

[52] L. Eberhardt, private communication.

[53] A. Arabi Ardehali, High-temperature asymptotics of supersymmetric partition functions, JHEP 07 (2016) 025 [arXiv: 1512.03376] [INSPIRE].

[54] T. Hartman, C.A. Keller and B. Stoica, Universal spectrum of $2 d$ conformal field theory in the large $c$ limit, JHEP 09 (2014) 118 [arXiv:1405.5137] [INSPIRE].

[55] N. Benjamin, M.C.N. Cheng, S. Kachru, G.W. Moore and N.M. Paquette, Elliptic genera and 3d gravity, Annales Henri Poincaré 17 (2016) 2623 [arXiv: 1503.04800] [INSPIRE].

[56] J.L. Cardy, Operator content and modular properties of higher dimensional conformal field theories, Nucl. Phys. B 366 (1991) 403 [INSPIRE].

[57] A. Arabi Ardehali, High-temperature asymptotics of the $4 d$ superconformal index, Ph.D. thesis, Michigan U., Ann Arbor, MI, U.S.A. (2016) [arXiv: 1605.06100] [InSPIRE].

[58] H.-B. Zhang and X. Zhang, One loop partition function from normal modes for $N=1$ supergravity in $A d S_{3}$, Class. Quant. Grav. 29 (2012) 145013 [arXiv:1205.3681] [inSPIRE].

[59] I.V. Tyutin and M.A. Vasiliev, Lagrangian formulation of irreducible massive fields of arbitrary spin in (2+1)-dimensions, Theor. Math. Phys. 113 (1997) 1244 [Teor. Mat. Fiz. 113N1 (1997) 45] [hep-th/9704132] [INSPIRE].

[60] A.N. Redlich, Gauge noninvariance and parity violation of three-dimensional fermions, Phys. Rev. Lett. 52 (1984) 18 [INSPIRE].

[61] A.N. Redlich, Parity violation and gauge noninvariance of the effective gauge field action in three-dimensions, Phys. Rev. D 29 (1984) 2366 [INSPIRE]. 